ARTICLE

https://doi.org/10.1038/s41467-019-12902-2

\title{
Increased yields and biological potency of knob- into-hole-based soluble MHC class II molecules
}

\author{
Pau Serra ${ }^{1 \star}$, Nahir Garabatos ${ }^{1}$ Santiswarup Singha ${ }^{2}$, César Fandos ${ }^{1}$, Josep Garnica ${ }^{1}$, Patricia Solé1, \\ Daniel Parras (1) 1, Jun Yamanouchi ${ }^{2}$, Jesús Blanco (1) 1,3, Meritxell Tort ${ }^{1}$, Mireia Ortega ${ }^{1}$, Yang Yang ${ }^{2,4}$,

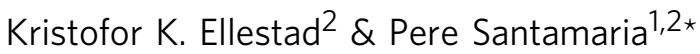

\begin{abstract}
Assembly of soluble peptide-major histocompatibility complex class II (pMHCII) monomers into multimeric structures enables the detection of antigen-specific $\mathrm{CD} 4^{+} \mathrm{T}$ cells in biological samples and, in some configurations, their reprogramming in vivo. Unfortunately, current $\mathrm{MHCII}-\alpha \beta$ chain heterodimerization strategies are typically associated with low production yields and require the use of foreign affinity tags for purification, precluding therapeutic applications in humans. Here, we show that fusion of peptide-tethered or empty MHCII- $\alpha \beta$ chains to the IgG1-Fc mutated to form knob-into-hole structures results in the assembly of highly stable pMHCII monomers. This design enables the expression and rapid purification of challenging $\mathrm{pMHCll}$ types at high yields without the need for leucine zippers and purification affinity tags. Importantly, this design increases the antigen-receptor signaling potency of multimerized derivatives useful for therapeutic applications and facilitates the detection and amplification of low-avidity $T$ cell specificities in biological samples using flow cytometry.
\end{abstract}

\footnotetext{
${ }^{1}$ Institut D'Investigacions Biomèdiques August Pi i Sunyer, Barcelona 08036, Spain. ${ }^{2}$ Julia McFarlane Diabetes Research Centre (JMDRC) and Department of Microbiology, Immunology and Infectious Diseases, Snyder Institute for Chronic Diseases and Hotchkiss Brain Institute, Cumming School of Medicine, University of Calgary, Alberta T2N 4N1, Canada. ${ }^{3}$ Division of Endocrinology, Hospital Clinic i Provincial de Barcelona, Barcelona, Spain. ${ }^{4}$ Department of Biochemistry and Molecular Biology, Cumming School of Medicine, University of Calgary, Alberta T2N 4N1, Canada. *email: pserra1@clinic.cat; psantama@ucalgary.ca
} 
Engineering and expression of soluble MHC molecules displaying specific antigenic epitopes (pMHC) has been instrumental not only for the analysis of antigen-specific $\mathrm{CD}^{+}$and $\mathrm{CD}^{+}{ }^{+}$-cell cells within biological samples ${ }^{1}$, but also for the production of pMHC-based compounds capable of modulating immune responses in vivo ${ }^{2-5}$.

Production of soluble pMHC class II molecules is more challenging than production of their pMHC class I counterparts. Assembly of pMHC class I monomers involves the heterodimerization of an MHC class I heavy chain with $\beta 2$ microglobulin via conserved, non-polymorphic residues in the presence of short (8-10 amino acid-long) peptides capable of binding to a peptide-binding groove located at the amino terminal end of the heavy chain. The rules that govern peptide binding to MHC class I molecules are well defined, including the identification of pockets capable of anchoring specific epitopes via complementary anchor residues that fix their binding register and stabilize the trimolecular complex. These structural hallmarks facilitate the expression and purification of stable pMHC class I monomers from eukaryotic expression systems, as well as the refolding and assembly of the trimolecular complex in vitro, using peptide and heavy and light chains expressed separately in prokaryotic expression systems.

Expression of MHC class II molecules has been significantly more difficult because secreted MHC class II $\alpha$ and $\beta$ chains lacking the transmembrane and cytoplasmic domains do not form stable heterodimers, even in the presence of high affinity peptide ligands. This is because the two $\alpha$-helical transmembrane domains of the MHC class II $\alpha$ and $\beta$ chains play key roles in the proper assembly and expression of stable heterodimers on the cell surface $^{6}$. This challenge was addressed by replacing the transmembrane and cytoplasmic domains of MHC class II chains by leucine zipper motifs (reviewed elsewhere ${ }^{7}$ ). However, since MHC class II binding peptides play a critical role in the assembly and stabilization of the $\alpha \beta$ heterodimer, these approaches do not invariably support the expression of pMHC monomers displaying epitopes with low affinity for MHC and/or the expression of MHC class II types with peculiar structural features, such as certain HLA-DQ molecules. This represents a fundamental limitation for the use of these reagents as a tool to enumerate and track cognate autoreactive T-cells in autoimmunity, where many naturally occurring autoimmune disease-relevant epitopes are weak MHC binders. This limitation is compounded by the fact that MHC class II (as opposed to class I) molecules can bind peptides of various lengths and on various registers through alternate anchor residues.

Another significant limitation of current soluble pMHC class II engineering approaches is that they are not currently suited for the production of multimeric pMHC class II-based compounds at scale for therapeutic purposes ${ }^{2,4,5}$. This is so because there are no orthogonal chromatographic separation schemes capable of purifying pMHC class II complexes from eukaryotic cell culture supernatants with the degree of purity, cost and yields required for clinical translation. Although for pure experimental purposes, this caveat can be addressed by addition of affinity separation tags into the pMHC complex, this practice is not acceptable for human translation as it bears the unacceptable risk of triggering the generation of anti-drug antibodies.

Here, we set out to engineer a generalizable molecular design capable of addressing these limitations. We report a new molecular pMHC class II heterodimerization design based on the "knob-into-hole" (KIH) approach used for the generation of bispecific monoclonal antibodies ${ }^{8,9}$. This approach allows stable alpha/beta heterodimerization for a broad range of MHC class II subtypes, with increased molecular stability, production yields and antigen-receptor binding and triggering potency. These compounds are also amenable to various modalities of signal amplification of multimer-based enumeration of cognate T-cells, and to the production of "empty" heterodimers suitable for peptide library screens.

\section{Results}

A mammalian expression system for soluble pMHC class II. We used lentiviral vectors encoding IRES-CFP or IRES-EGFP reporter cassettes (Fig. 1a) to express pMHCIIs in CHO cells. The pMHCII $\alpha$ and $\beta$ chains were either transcribed from a single ORF as two chains separated by a P2A ribosomal skipping sequence (Fig. 1b), or from two different ORFs in different vectors (Fig. 1c). Figures 2-4 summarize the structural features of representative constructs for the various pMHCIIs described here, as well as key junctional sequences. Table 1 provides a list of the

a

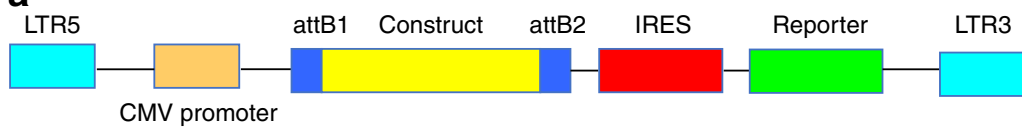

b

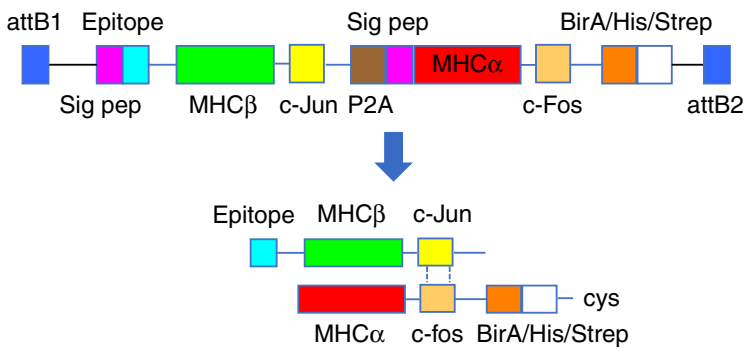

C

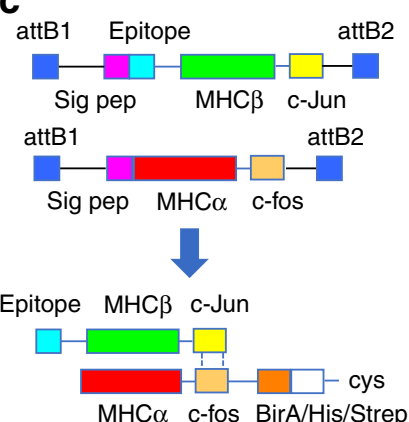

Fig. 1 pMHCII-expression construct design. Cartoons depict the general structure of the lentiviral system (a) and the type of constructs used (b, c). b Structure of a P2A-linked $\mathrm{pMHC} \beta$ and $\mathrm{MHC} \alpha$ chain-coding construct (top) and a representation of the resulting pMHCII product secreted into the cell culture supernatant (bottom). c Single $\mathrm{pMHC} \beta$ - or $\mathrm{MHC} \alpha$ chain constructs that were serially transduced into $\mathrm{CHO}$ cells to produce the resulting pMHCII $\alpha \beta$ heterodimers (bottom) 


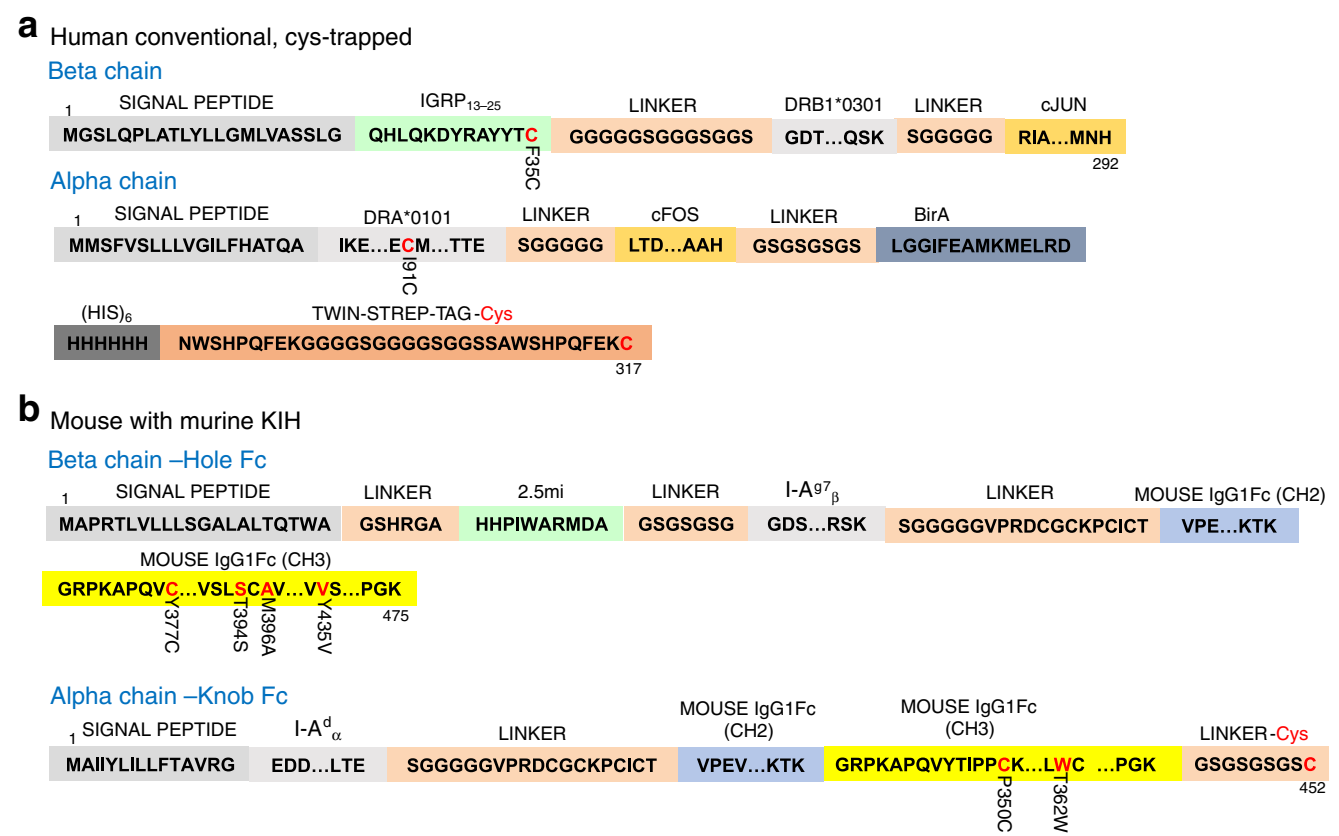

Fig. 2 Key junctional, linker and motif sequences for cys-trapped human pMHCII and mKIH-based mouse pMHCII. a Key amino acid sequences of human pMHCII molecules encoding a cys-trapped IGRP ${ }_{13-25} / \mathrm{DRA} 1^{\star} 0101 / \mathrm{DRB1}{ }^{\star} 0301 \mathrm{pMHCII}$ heterodimerized via c-jun/c-fos leucine zippers (also referred to "conventional"). '..' is used to indicate that corresponding intervening amino acid sequences are not shown, as they are publicly available. Residues in red are mutated and the original residue and its position are indicated immediately below. $\mathbf{b}$ Key amino acid sequences of a mouse pMHCII molecule encoding $\mathrm{BDC} 2.5 \mathrm{mi} / \mathrm{IA} \alpha^{\mathrm{d}} / \mathrm{IA} \beta^{g 7}$ heterodimerized using a carboxyterminal mouse IgG1-Fc-based $\mathrm{KIH}$

mouse and human pMHCIIs used here and their expression yields. Briefly, transduced CHO-S cells expressing high levels of EGFP and CFP were sorted by flow cytometry and grown in protein-free media in shake flasks using a fed batch protocol. pMHCIIs were purified from supernatants and used directly to coat iron oxide nanoparticles (NPs), or were biotinylated to produce pMHCII tetramers.

Leucine zipper-based pMHCII have variable stability. It has been shown that the epitope is a major stabilizer of soluble pMHCII heterodimers ${ }^{10,11}$. Peptides binding with high affinity support higher heterodimer stability than those binding with low affinity. However, intrinsic molecular properties of allelic MHCII molecules also play a major role in defining the stability of pMHCIIs, independently of the peptide ${ }^{12,13}$. As a result, whereas certain pMHCII molecules migrate as a single, large molecular species in non-denaturing SDS-PAGE, most others melt into single $\alpha$ and $\beta$ chains (Fig. 5a) and are expressed at low yields (Table 1).

Cys-trapped pMHCII heterodimers increase pMHC stability. We reasoned that we could increase the stability and possibly the production yields of SDS-unstable c-jun/c-fos-zippered MHCIIs (herein also referred to 'conventional') by introducing cysteines at appropriate positions in the peptide and the MHCII $\alpha$ chain to anchor the peptide onto the MHC on a preferred binding register ${ }^{14,15}$ (herein referred to as cys-trapping (CT)). We note that our prior attempts to address this issue by introducing artificial disulfide bonds at or near the c-jun/c-fos zipper in poorly expressing pMHCII constructs were unsuccessful. We first focused on the type 1 diabetes (T1D)-relevant IGRP $_{13-25} /$ DRB1 ${ }^{*} 0301 / D R A 1 * 0101$ complex (Table 1 ). We replaced a Cterminal phenylalanine in IGRP I3-25 $_{13}$ and a proximal serine in the MHCII a chain for cysteines (Figs. 2-4). This resulted in SDS stability (Fig. 5b) without any appreciable loss of cognate T-cell binding efficiency, as measured using pMHC tetramers and a human CD4/TCR-transduced Jurkat cell line (Fig. 5c). Similar results were obtained with other pHLA molecules, such as IGRP $_{23-35} / \mathrm{DRB}^{*}{ }^{*} 401 / \mathrm{DRA} 1^{\star} 0101$ (Fig. $5 \mathrm{~b}$ ). The use of a cystrap also enabled the production of much more difficult-toexpress HLA molecules, such as HLA-DQB1 ${ }^{*} 0201 / \mathrm{DQA1}{ }^{\star} 0501$ displaying gliadin residues 62-72 (Table 1 and Fig. 5b). Cystrapping, however, increased production yields for some but not all pMHCs (e.g., IGRP ${ }_{13-25} / \mathrm{DRB1}^{*} 0301 / \mathrm{DRA}^{*} 0101$ ) (Table 1 ). Furthermore, cys-trapping cannot be adopted by all pMHCIIs, because introduction of artificial cysteines within the peptide might in some cases impair T-cell binding and/or activation, and because epitopes that already contain naturally occurring cysteines within their sequence are not suitable for this approach.

A knob-into-hole-based pMHCII design. To address this and other limitations of current pMHCII production strategies, including heterodimer instability, discrete production yields, and the lack of efficient and scalable purification schemes broadly applicable to any pMHC type (for human in vivo use), we explored the feasibility of using a knob-into-hole (KIH)-IgGbased heterodimerization strategy. Introduction of complementary amino acid substitutions in the $\mathrm{CH} 3$ domain of the $\mathrm{Fc}$ region of human IgG1 (or other IgG subtypes) results in the generation of two different $\mathrm{Fc}$ molecules (knob and hole) with favorable heterodimerization and unfavorable homodimerization potential ${ }^{8,9}$. We reasoned that, unlike $\mathrm{Fc}$-fusion-based pMHC dimerization, which generates large Ig-like molecular structures in which $\alpha \beta$ heterodimer formation and stability still require the use of leucine zippers and are regulated by the same principles that control the assembly of non-Fc-fused, c-jun/c-fos zippered pMHCIIs ${ }^{16-19}$, KIH-based pMHCII heterodimerization would potentially render pMHCIIs intrinsically more stable with only a relatively minor increase in total molecular weight.

We tethered the mouse $I A \alpha^{\mathrm{d}}$ chain with a modified $\mathrm{Fc}$ region of human IgG1 to behave as a knob (both with and without the cfos motif), and the corresponding IA $\beta^{\mathrm{g} 7}$ chain (with and without 
a

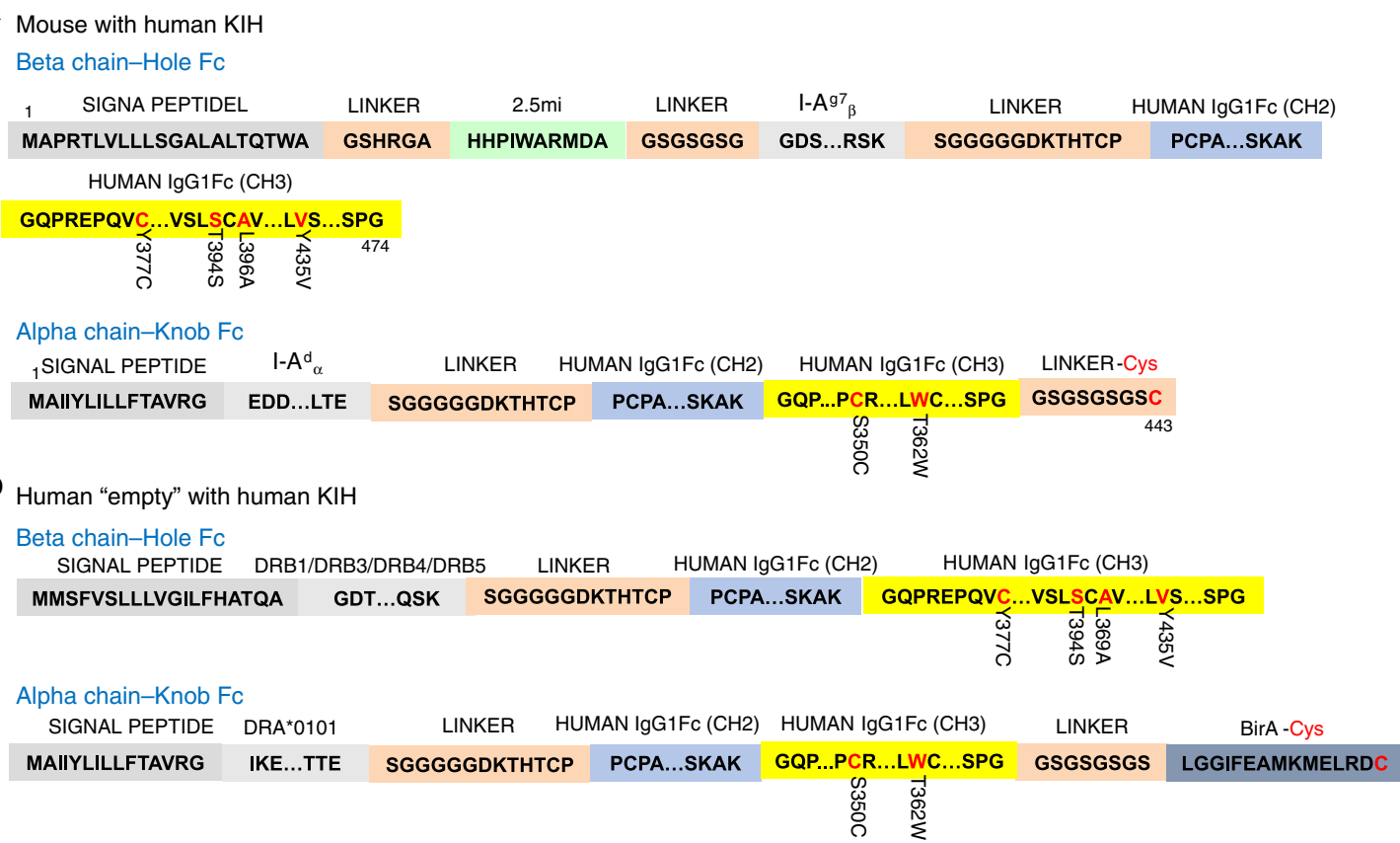

Fig. 3 Key junctional, linker and motif sequences for hKIH-based mouse pMHCII and empty hKIH-based human pMHCII. a Key amino acid sequences of a mouse pMHCII molecule encoding BDC2.5mi//A $\alpha^{\mathrm{d}} / \mathrm{IA} \beta^{\mathrm{g} 7}$ heterodimerized using a carboxyterminal human lgG1-Fc-based $\mathrm{KIH}$. b Key amino acid sequences for "empty" human MHCll molecules encoding DRA 0101 MHC $\alpha$ and DRB1, DRB3, DRB4 or DRB5 MHC $\beta$ chains heterodimerized using a carboxyterminal human IgG1-Fc-based KIH

the $\mathrm{c}$-jun motif) to the $\mathrm{Fc}$ region of human IgG1 modified to behave as a hole (Figs. $3 \mathrm{a}$ and $6 \mathrm{a}, \mathrm{b})$. In our initial designs, we also included a BirA biotinylation site, a $6 \times$ histidine and twin strep tags, and a cysteine at the C-terminal end of the knob, generating a 'knob' that is larger than its 'hole' counterpart. Both the leucinezippered and non-zippered cell lines expressed the transgenic RNA, as documented by the expression of EGFP (Fig. 6c, left), but only the latter secreted protein G-binding material in the supernatant (Fig. $6 c$, right), which ran as a single band in native SDS-PAGE (Fig. 6d, left panel), and as two separate bands of different molecular weight, as expected, but similar intensity in denaturing SDS-PAGE (Fig. 6d, right panel), suggesting $~ 1: 1$ stoichiometries. The KIH version of this pMHCII expressed at $>4$ fold higher levels than its non-KIH-based counterpart (Table 1). These molecules folded appropriately because pMHCII tetramers generated with these KIH-based pMHCII monomers stained splenic $\mathrm{CD}^{+}{ }^{+} \mathrm{T}$-cells from a transgenic mouse expressing a BDC2.5mi-specific T-cell receptor (TCR) essentially like its zippered, non-KIH-based counterpart (Fig. 6e).

Receptor-signaling properties of hIgG1 KIH-based pMHCII. When delivered systemically, NPs coated with autoimmune disease-relevant pMHCII (pMHC-NP) can reprogramme (and expand) autoantigen-experienced effector/memory $\mathrm{T}$-cells into cognate T-regulatory type 1 (TR1) cells, leading to reversal of various autoimmune diseases ${ }^{2,5}$. The biological potency of these compounds (TR1 cell formation in vivo) is a function of pMHC valency on the NP surface and can be gauged in vitro using reporter cell lines 4 .

We coupled these molecules to maleimide-functionalized NPs via their C-terminal free cysteine as described in ref. ${ }^{4}$. As shown in Fig. 7a, most of the pMHC in the preparations remained coupled to the NPs when electrophoresed in SDS-PAGE under native conditions, but were released (as NP-free PEG-pMHC conjugates) under denaturing conditions. Quantification of the pMHC valencies of these compounds indicated that, in general, these $\sim 50 \%$ larger $\mathrm{KIH}$-based pMHC monomeric structures coat at lower valencies ( $20 \%$ fewer) than their conventional non$\mathrm{KIH}$-based counterparts $(45 \pm 2.8$ vs. $36 \pm 2.5 ; n=4$ and 5 , respectively).

We next compared the TCR signaling potency of NPs coated with non-KIH-based BDC2.5mi/IA' 7 pMHC (at $65 \mathrm{pMHCs} / \mathrm{NP}$ ) with NPs coated with its KIH-based counterpart (at 37 pMHCs/ NP) (Fig. 7a), on Jurkat cells co-expressing mouse CD4, a cognate TCR and NFAT-driven luciferase. Both compounds had similar potency, despite carrying significantly different $\mathrm{pMHC}$ valencies (Figs. 7b and $8 \mathrm{a}, \mathrm{b}$ ). This suggested that these KIH-based pMHCNP structures might perform optimally at pMHC valencies falling below the minimal optimal pMHC valencies defined for the conventional pMHC design ${ }^{4}$.

In vivo biological activity of hIgG1 KIH-based pMHCII-NPs. Having shown that pMHC-NPs produced using KIH-based pMHCs have adequate TCR-binding and signaling potency, we sought to confirm that these compounds could also trigger the formation and expansion of cognate TR1 cells in vivo, as is the case for compounds produced using conventional pMHCs. As shown in Fig. 7c, the KIH-based pMHCII-NP compounds triggered the formation and expansion of similar numbers of cognate (tetramer ${ }^{+}$) TR1 cells as their non-KIH-based counterparts. Furthermore, the cognate (tetramer ${ }^{+}$) CD4+ T-cells expanding in vivo in response to both types of compounds secreted TR1-relevant cytokines upon stimulation with anti-CD3 and antiCD28 mAbs ex vivo, as compared with their tetramer ${ }^{-} \mathrm{CD}^{+}$ T-cells (Fig. 7d). Thus, these zipper-less KIH-based pMHCs heterodimers have similar biological activity than the zippered conventional pMHC.

Biological in vitro properties of mIgG1 KIH-based pMHCII. The heterodimerization potential of a mouse IgG1-based $\mathrm{KIH}$ structure has not been previously described. We thus explored the possibility of generating BDC2.5-I-A $\beta^{\mathrm{g} 7}-\mathrm{Hole} / \mathrm{I}-\mathrm{A} \alpha^{\mathrm{d}}-\mathrm{Knob}$ 


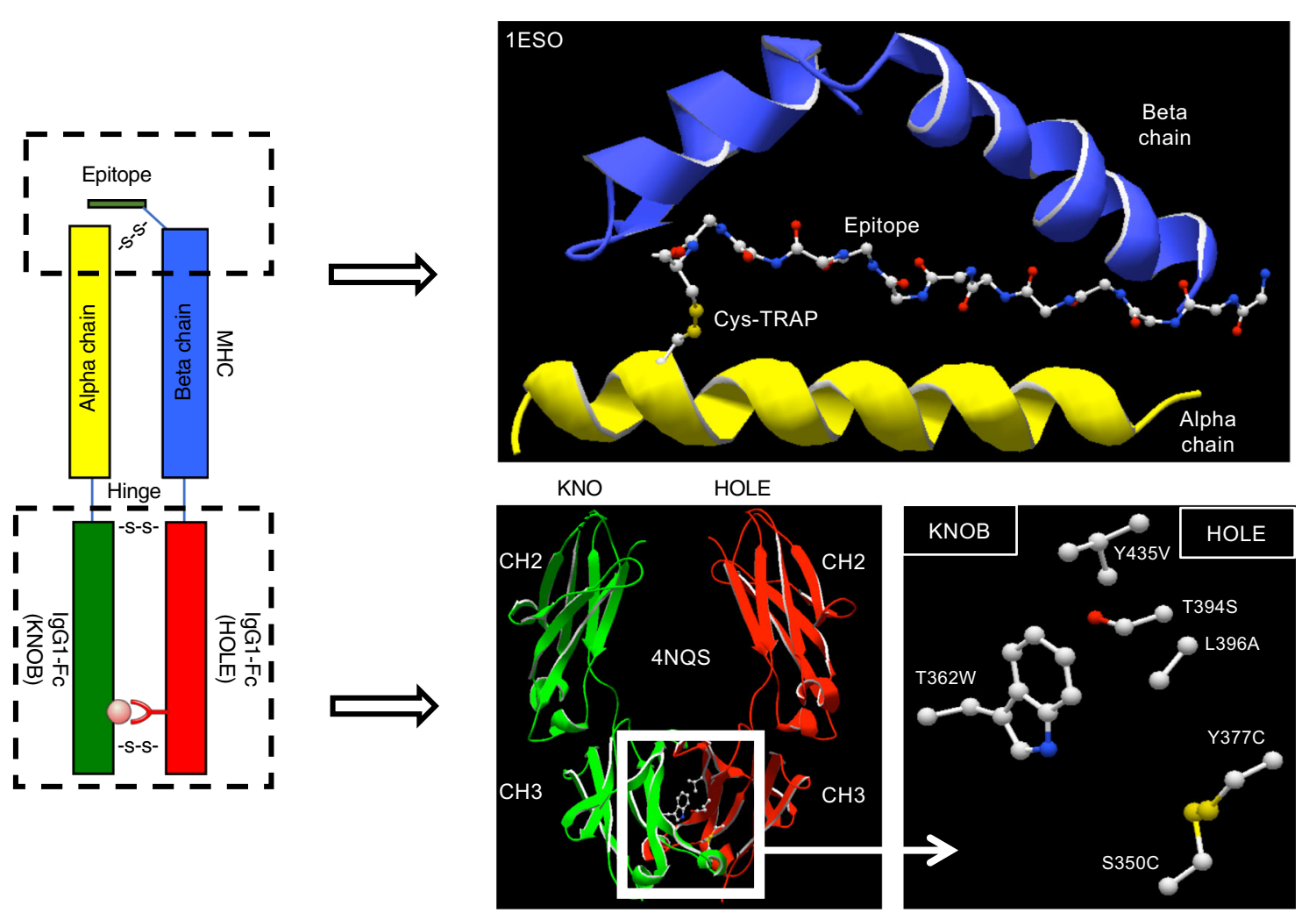

Fig. 4 Cartoons depicting the structure of the $\mathrm{KIH}$-based pMHCII constructs or specific domains. Left: primary structure of a Cys-trapped $\mathrm{KIH}$-based pMHCII heterodimer. Top right: secondary structure of the peptide-binding domain loaded with a peptide bound to the MHCII molecule on a specific register via a disulfide bridge between the carboxyterminal end of the peptide and a complementary Cys on the MHCII $\alpha$ chain. Bottom right: predicted quaternary structure of the $\mathrm{KIH} \mathrm{Fc} \mathrm{portion} \mathrm{of} \mathrm{the} \mathrm{KIH}$-based constructs and the key amino acid substitutions that were used to promote $\mathrm{KIH}$-based heterodimerization

heterodimers in which the knob-Fc and hole-Fc are derived from the mIgG1-Fc sequence ( $\mathrm{mKnob}$ and mHole, respectively). This mouse KIH approach would help reduce the unwanted immunogenicity of these compounds used for in vivo experimentation in mice. To this end, we identified the mouse residues that could generate functional mKnob and mHole molecules upon modification (Fig. 2b). The banding patterns of the protein G-purified molecules in denaturing SDS-PAGE gels was essentially identical to those seen for their hIgG1 KIH-based counterparts. In addition, NPs coated with these molecules had quantitatively similar antigen-receptor-signaling properties as NPs coated with their hIgG1 KIH-based counterparts (Figs. 7e and 8c, d).

Production of hIgG1 KIH-based human pMHCII. We next asked if this $\mathrm{KIH}$ strategy could also be used to stabilize weaker peptide:MHC interactions, such as $\operatorname{IGRP}_{13-25} / \mathrm{DRB}^{\star} 0301 /$ DRA $1^{\star} 0101$. As was the case for zippered BDC2.5-I-A $\beta$ g7-Hole/I$\mathrm{A} \alpha^{\mathrm{d}}$-Knob heterodimers, zippered IGRP ${ }_{13-25}-\mathrm{DRB1}^{\star} 0301$-Hole/ DRA $1^{\star} 0101-K n o b$ heterodimers could not be expressed, but removal of the c-jun/c-fos zipper from the molecule led to efficient expression, at levels significantly greater than those obtained from CHO-S cells secreting non-KIH-based IGRP $_{13-25^{-}}$ DRB $1^{\star} 0301 / \mathrm{DRA} 1^{\star} 0101$ heterodimers (Table 1 ). pMHCII tetramers produced with the $\mathrm{KIH}$-based monomers stained cognate $\mathrm{T}$ cells essentially like tetramers produced using leucine-zippered pMHCII monomers (Fig. 9a). Addition of the cys-trap register fixing mutations in the peptide and MHCII a chain of these complexes (Figs. 2a and 4) further increased expression yields (Table 1). This molecular modification did not disrupt the TCRbinding properties of these molecules because staining of cognate TCR-expressing Jurkat cells with tetramers made with the CT vs
non-CT KIH-based constructs was essentially equivalent (Fig. 9b). Furthermore, these molecules reacted quantitatively equally to an anti-DR mAb (clone L243) that binds to a conformational epitope on the HLA-DRa chain that requires the correct folding of the $\alpha \beta$ heterodimer ${ }^{20,21}$ (Fig. 9c).

Cys-trapping augments the potency of KIH-based pMHCII. The above data suggested that introduction of a cys-trap between IGRP $_{13-25}$ and DR3 increases the structural stability of the heterodimer and $\mathrm{pMHC}$ production yields without interfering with TCR binding. However, when we compared the in vitro potency of NPs coated with a cys-trapped version of the non-KIH-based human $\mathrm{IGRP}_{13-25} / \mathrm{DRB}^{\star} 0301-\mathrm{DRA}^{\star} 0101 \mathrm{pMHC}$ (at $63 \mathrm{pMHCs} /$ NP) with that of NPs coated with three non-cys-trapped $\mathrm{KIH}$ based IGRP ${ }_{13-25} / \mathrm{DRB}^{\star} 0301-\mathrm{DRA}^{\star} 0101$ preparations (at 46, 29, and $27 \mathrm{pMHCs} / \mathrm{NP}$ ), the latter three elicited significantly reduced luciferase responses from cognate Jurkat cells (Figs. 8e, f and 9d). The following three lines of evidence suggested the possibility that these differences might be accounted for by the presence of the cys-trap in the non-KIH-based pMHC that was used as a control. First, NP preparations displaying low valencies of the KIH-based BDC2.5mi/IAg7 pMHC performed essentially like NPs displaying high valencies of its zippered, non-KIH-based counterpart (Figs. 2b and $8 \mathrm{a}, \mathrm{b}$ ), suggesting that KIH-based pMHCs support increased TCR signaling. Second, all three NP preparations displaying the non-cys-trapped $\mathrm{KIH}$-based $\mathrm{IGRP}_{13-25} / \mathrm{DRB}^{\star} 0301$ $\mathrm{DRA}^{*} 0101$ also performed similarly in this assay, in a valencyindependent manner (from 27-46 pMHCs/NP), consistent with the hypothetical increased potency of KIH-based designs.

To investigate this hypothesis, we compared the biological potency of NPs coated with cys-trapped and non-cys-trapped 
Table 1 Peptides, MHC molecules, heterodimerization domains and yields

\begin{tabular}{|c|c|c|c|c|c|}
\hline Tethered epitope & Sequence & MHC beta & MHC alpha & Heterodimers & Yield (mg/L) \\
\hline BDC.2.5mi & HHPIWARMDA & $1-A_{\beta}{ }^{g 7}$ & $1-A_{\alpha}{ }^{d}$ & JUN/FOS & 17.7 \\
\hline $\mathrm{BDC} .2 .5 \mathrm{mi}$ & HHPIWARMDA & $1-A_{\beta} g 7$ & $1-A_{\alpha} d$ & HOLE/KNOB & 80.6 \\
\hline TOPO (722-736) & KLNYLDPRITVAWCK & $1-A_{\beta} b$ & $1-A_{\alpha}^{b}$ & JUN/FOS & 2.3 \\
\hline mDSG3(301-315) & RNKAEFHQSVISQYR & $1-A_{\beta}^{p} b$ & $1-A_{\alpha} b$ & JUN/FOS & 0.2 \\
\hline IGRP(13-25) & QHLQKDYRAYYTF & DRB1*0301 & $\mathrm{DRA}^{\star} 0101$ & JUN/FOS & 12 \\
\hline IGRP(13-25) cys-trap & QHLQKDYRAYYTC & DRB1*0301 & $\mathrm{DRA}^{\star} 0101$ & JUN/FOS & 4 \\
\hline PPI(76-90)88S cys-trap & SLQPLALEGSLQSRC & $\mathrm{DRB} 1{ }^{\star} 0401$ & $\mathrm{DRA}^{\star} 0101$ & JUN/FOS & 4.12 \\
\hline $\operatorname{PPI}(76-90) 88 \mathrm{~S}$ & SLQPLALEGSLQSRG & $\mathrm{DRB} 1^{\star} 0401$ & $\mathrm{DRA}^{\star} 0101$ & HOLE/KNOB & 44.5 \\
\hline IGRP(23-35) & YTFLNFMSNVGDP & $\mathrm{DRB} 1^{\star} 0401$ & $\mathrm{DRA}^{\star} 0101$ & JUN/FOS & 0.6 \\
\hline IGRP(23-35) cys-trap & YTFLNFMSNVGDC & $\mathrm{DRB} 1^{\star} 0401$ & $\mathrm{DRA}^{\star} 0101$ & JUN/FOS & 45.9 \\
\hline Glia(62-72) cys-trap & PQPELPYPQPC & DQB1*0201 & DQA1*0501 & JUN/FOS & 2.6 \\
\hline Glia(62-72) & PQPELPYPQPE & DQB1*0201 & DQA1*0501 & HOLE/KNOB & 30.4 \\
\hline Glia(62-72) cys-trap & PQPELPYPQPC & DQB1*0201 & DQA1*0501 & $\mathrm{HOLE} / \mathrm{KNOB}$ & 39.5 \\
\hline
\end{tabular}

This Table was generated using contemporary $\mathrm{CHO}$ cell cultures using representative cell lines

versions of both types of pMHC constructs (non-KIH-based, and $\mathrm{KIH}$-based). Surprisingly, with both construct types, inclusion of a cys-trap boosted potency (Figs. 8g, h and 9e). Indeed, NPs coated with the cys-trapped KIH-based construct had similar function as NPs coated with the cys-trapped non-KIH-based construct, despite significant differences in pMHC valency (56 and 63 for cys-trapped and non-cys-trapped non-KIH-based pMHC, respectively, vs. 25 and 26 for cys-trapped and non-cystrapped $\mathrm{KIH}$-based pMHC, respectively), again supporting the idea that the use of KIH-based pMHCs on NPs lowers the pMHC valency threshold required for biological activity.

Together, these unexpected observations suggest that the peptide-binding cleft in some non-cys-trapped pMHC monomers (whether leucine zipper (conventional) or KIH-based) might be occupied by endogenous CHO-S peptides, as opposed to the tethered peptides encoded in the expression constructs. Alternatively, this approach generates a more compact pMHC structure that somehow enhances cognate TCR engagement. The increased biological potency of the KIH-based design, particularly when combined with peptide cys-trapping, suggest the possibility that the KIH halve of these pMHC class II molecules may have an allosteric effect on the pMHC interface in a way that improves TCR engagement. Alternatively, this effect might be due to the particular topology adopted by these KIH-based molecules on NPs. The radii of the KIH-based pMHCs on NPs is larger than those of their zippered counterparts. This may decrease the occurrence of potentially disruptive interactions of the TCRbinding N-terminal pMHC domain with the NP during the functionalization process. In this regard, the $\mathrm{KIH}$ portion of the KIH-based pMHC molecule might buffer conformational changes on the pMHC's N-terminal domain imposed by the NP. In addition, the disulfide bonds that are introduced into these KIHs might contribute to the hypothetical resistance of these molecules to allosteric changes induced by neighboring pMHC molecules or the NP itself. Whatever the precise explanation, at a practical level, these molecular modifications actually change the minimal optimal pMHC density threshold for pharmacodynamic activity in vivo; the " 32 " minimal optimal pMHC valency threshold defined for 20-nm diameter NPs displaying conventional pMHCs appears to be lower for Cys-trapped, KIH-based pMHCs ${ }^{4}$.
hIgG1 KIH-based stabilization of peptide-HLA-DQ molecules. Peptide-HLA-DQ complexes are difficult to express ${ }^{22}$. As noted above, we could only produce significant amounts of c-jun/c-foszippered Gliadin ${ }_{62-72} / \mathrm{DQB} 1^{\star} 0201 / \mathrm{DQA} 1^{\star} 0501$ when the peptide was cys-trapped onto the MHC molecule, albeit at low yields (Table 1 and Fig. 5b). Remarkably, substitution of the leucine zipper domain for a $\mathrm{KIH}$ enabled the production of Gliadin $62-72 /$ DQB $1^{\star} 0201 / D Q A{ }^{\star} 0501$ by $\mathrm{CHO}-\mathrm{S}$ cells at yields that were 15 fold higher (Table 1 and Fig. 9f).

KIH-based stabilization of empty MHC class II molecules. Some experimental approaches for T-cell epitope mapping require the use of extensive arrays of pMHCII tetramers to identify epitope reactivity by flow cytometry ${ }^{23-25}$. In this context, the use of pMHCII molecules displaying covalently tethered peptides is not practical, as it implies purifying many different pMHCII molecules and generating the corresponding fluorochrome-labeled tetramers for each specific epitope. We thus investigated if the $\mathrm{KIH}$-based approach could also be used to express high levels of non-peptide-tethered pMHCIIs from $\mathrm{CHO}$ cells and whether these compounds could be used for peptideloading in vitro ${ }^{26,27}$. As shown in Table 1, transduced CHO-S cells secreted high levels of four different non-peptide-tethered human DRB types, including $\mathrm{DRB} 1^{\star} 0301 / \mathrm{DRA} 1^{\star} 0101, \mathrm{DRB} 4$ ${ }^{\star} 0101 / \mathrm{DRA}^{\star} 0101, \quad \mathrm{DRB}^{\star}{ }^{\star} 0101 / \mathrm{DRA}^{\star} 0101$, and $\mathrm{DRB} 1^{\star} 1501 /$ $\mathrm{DRA}^{\star} 0101$. Importantly, these complexes could be loaded with peptides in vitro and the corresponding tetramers bound to cognate T-cells essentially like their peptide-tethered counterparts (Fig. 9g, h).

Amplification of KIH-based pMHCII multimer staining. Different strategies have been described to increase the staining intensity cognate T-cells with fluorochrome-labeled pMHC multimers ${ }^{28}$, including the use of kinase inhibitors, the formation of cooperative pMHC/TCR clusters with crosslinking antibodies $^{29}$, and the use of scaffolds enabling the production of higher-order multimeric structures such as dextramers ${ }^{30}$. This is particularly useful in autoimmune diseases, where the peripheral frequencies of autoreactive T-cells and their avidity for cognate 
a

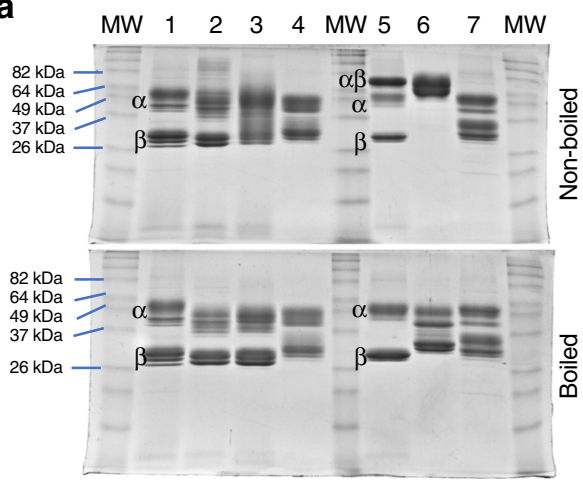

C

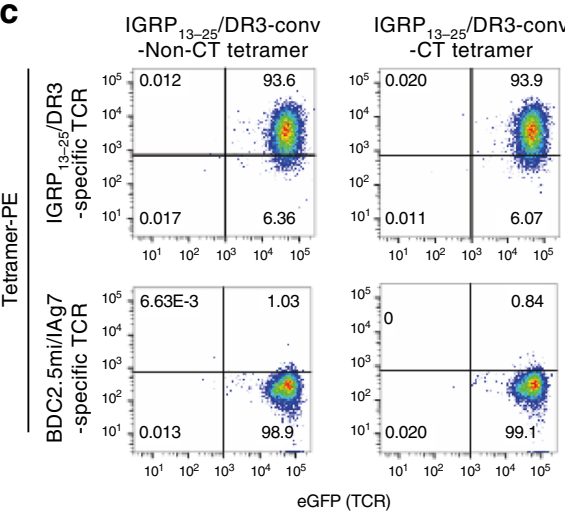

b

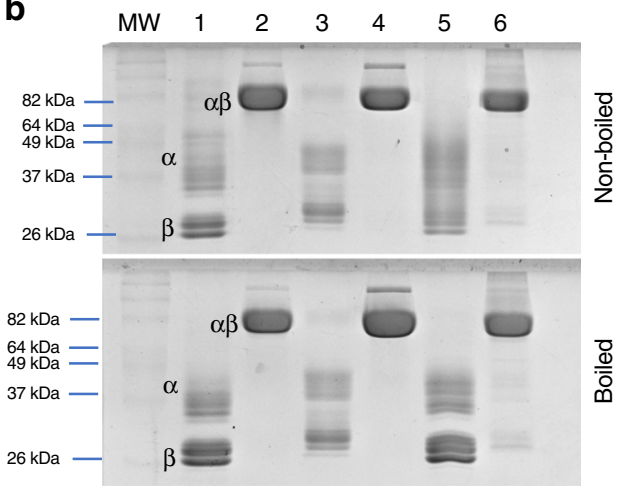

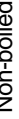

$\frac{\bar{Q}}{\overline{0}}$

Fig. 5 Stabilization of pMHCII heterodimers by introduction of peptide-MHC $\alpha$ chain Cys-traps. a SDS-PAGE for various c-jun/c-fos-based pMHCII

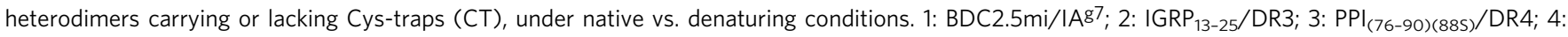

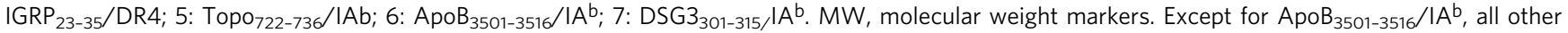
pMHCII heterodimers shown are partially or completely SDS unstable. The electrophoretic behaviors of the various pMHCs shown herein were consistent with those seen with earlier preparations of the same proteins that had been previously run in separate gels at least once before. They were re-expressed, re-purified and re-run again together to generate this figure. $\mathbf{b}$ Effects of Cys-trapping on SDS stability of pMHCII heterodimers. Data correspond to 1: $I_{\text {IGRP }}$ 13-25/DR3-non-CT; 2: IGRP 13-25/DR3-CT; 3: IGRP 23-35/DR4-non-CT; 4: IGRP 23-35/DR4-CT; 5: PPI 76-90(88s)/DR4-non-CT; and 6: Glia $62-72 / D Q 2-C T$. c pMHCIl tetramer/CD4 FACS dot plots for Jurkat cells expressing human CD4 and an IGRP $13-25$ /DR3-specific TCR (top) or mouse CD4 and a BDC2.5mi/ $I^{g}{ }^{7}$-specific TCR (bottom) stained with non-CT (left) or CT (right) IGRP $_{13-25} / D R 3$ tetramers. The staining patterns shown are representative of at least two independent experiments

pMHC complexes are significantly lower than those seen for foreign antigen-specific T-cells, such as in the context of infection and allergy. We thus investigated whether the signal-to-noise ratio of cognate T-cell staining with pMHCII tetramers could be improved using anti-hIgG-based amplification of KIH-based pMHCII tetramer binding. Human PBMCs were spiked with human clonal $\mathrm{IGRP}_{13-25} / \mathrm{DR} 3$-specific $\mathrm{CD}^{+}{ }^{+} \mathrm{T}$-cells and stained with cognate $\mathrm{KIH}$-based pMHCII tetramers in the presence or absence of the protein kinase inhibitor Dasatinib (to inhibit TCR downregulation) followed by anti-hIgG-PE amplification. As shown in Fig. 9i, anti-hIgG increased the mean fluorescence signal intensity of tetramer staining, both in the presence and absence of Dasatinib.

\section{Discussion}

We have described a novel pMHCII heterodimerization strategy that enables the production and purification, at high yields, of stable pMHCII monomers for a variety of applications. In this novel approach, we replace the transmembrane and cytoplasmic regions of the MHCII $\alpha$ and $\beta$ chains for the human or mouse IgG1-Fc modified to form knobs and holes, respectively. These molecules are invariably SDS stable, express at significantly higher levels than conventional leucine-zippered pMHCIIs and can be easily purified from culture supernatants using protein A/G chromatography without the need to include foreign, immunogenic affinity-purification tags in the molecule. In addition, they have superior TCR binding and triggering properties and, when used as multimeric structures to enumerate antigen-specific T-cells in complex biological samples, are amenable to signal amplification, including the use of anti-hFc antibodies. Collectively, the advantages of the molecular pMHCII engineering approach described herein overcome the roadblocks that currently preclude the use of pMHCII designs for therapeutic applications. In addition, the high expression yields of non-peptide-tethered KIH-based pMHCs should facilitate the screening of epitope libraries in the context of specific MHCII molecules and antigen receptors. This $\mathrm{KIH}$ approach could also help stabilize difficult-to-express soluble TCR $\alpha \beta$ heterodimers for multiple uses, including the identification of specific pMHC targets $^{31}$.

\section{Methods}

Mice. NOD/Lt and BDC2.5-NOD mice (expressing a transgenic T-cell receptor for the BDC2.5mi//A ${ }^{77}$ complex) ${ }^{32}$ were purchased from the Jackson Lab (Bar Harbor, ME). Mice were housed and bred in a specific pathogen-free facility at the Cumming School of Medicine at the University of Calgary. The animal experiments described herein were approved by the University of Calgary Animal Care Committee and complied with the Canadian Council of Animal Care.

pMHC production. Recombinant pMHCII were produced in CHO-S cells (Invitrogen) transduced with lentiviruses (Vector Builder, Chicago, IL) encoding a monocistronic message in which the peptide-MHC $\beta$ (or non-peptide-tethered 
a

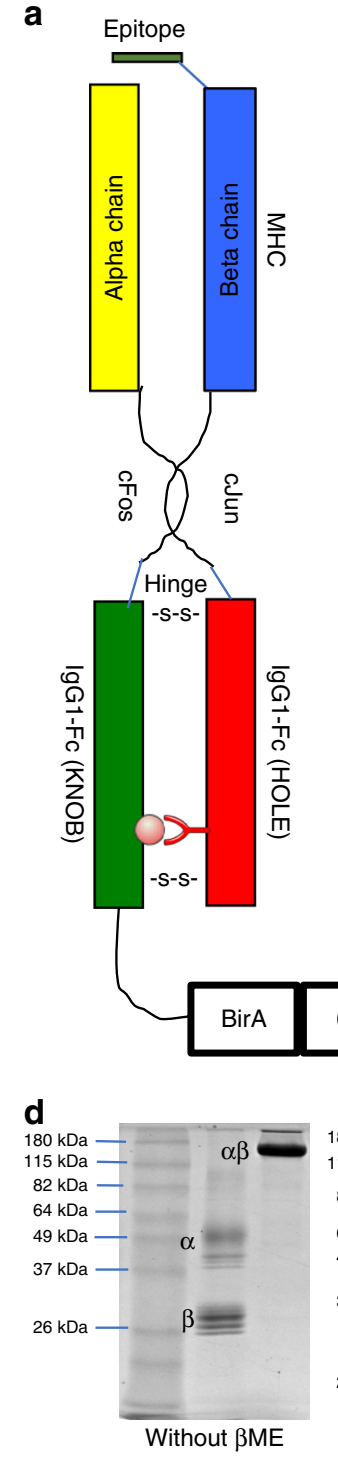

b

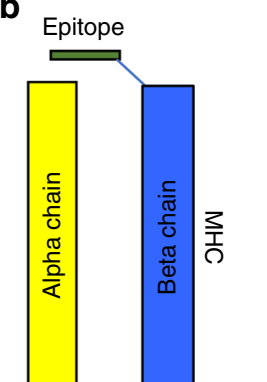

C
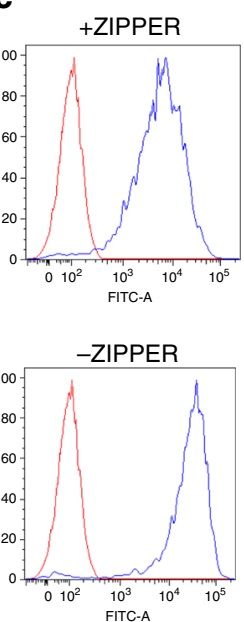
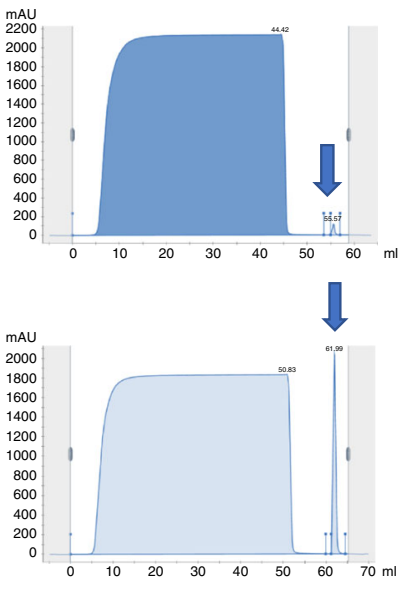

$6 \mathrm{xHis}$

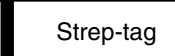
Cys

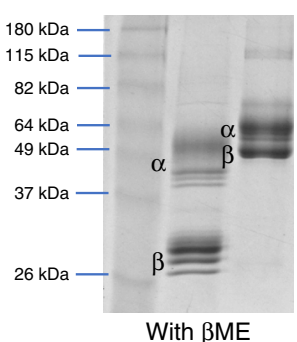

e
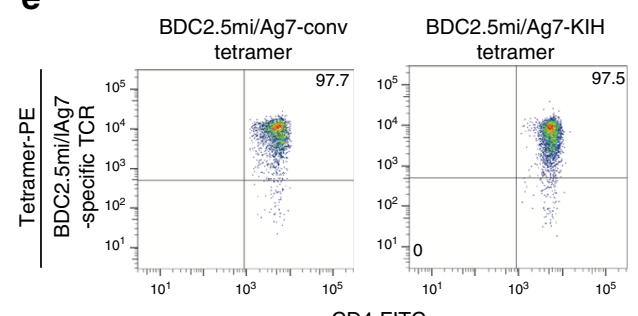

CD4-FITC

Fig. 6 Use of a c-jun/c-fos zipper in $\mathrm{KIH}$-based pMHCII is incompatible with expression. $\mathbf{a}$, b Cartoons displaying the structure of the two types of $\mathrm{KIH}$ constructs tested here. c (left), Expression of eGFP in CHO-S cell lines transduced with lentiviruses encoding the constructs depicted in A (top) and B (bottom), indicating adequate construct transcription and translation. c (right), FPLC elution profiles of pMHC class II from Strep-tactin columns loaded with supernatants from $\mathrm{CHO}$ cells expressing the constructs in A (top) or B (bottom). $\mathbf{d}$ Effects of the KIH on the SDS stability of a representative pMHCII heterodimer (out of at least 10 different $\mathrm{KIH}$-based pMHCs), in the absence of Cys-trapping. Data correspond to c-jun/c-fos-based BDC2.5mi/IAg7 ('conv', left lane) and a KIH-based BDC2.5mi/IAg7 (right lane). $\beta M E$, beta-mercaptoethanol. e Representative pMHCIl tetramer/eGFP (TCR) FACS dot plots for BDC2.5-TCR-transgenic CD4 ${ }^{+}$T-cells stained with c-jun/c-fos- ('conv', left) or KIH-based BDC2.5mi/IAg7 tetramers (right). The staining patterns shown are representative of at least two independent experiments

MHC $\beta$ ) and MHCa chains were separated by a ribosome skipping P2A-coding sequence, followed by an IRES-EGFP cassette ${ }^{33}$. Alternatively, the peptide-MHC $\beta$ and MHC $\alpha$ chains were encoded in separate lentiviruses encoding IRES-EGFP and IRES-CFP cassettes, respectively. Peptide-tethered MHCII molecules were biotinylated in vitro, as described below. "Empty" MHCII molecules were biotinylated in vivo, by expressing the corresponding lentivirus-transduced constructs in BirAtransgenic $\mathrm{CHO}$ cells (see below).

To express the various pMHCIIs, transduced CHO-S cells were grown in $2 \mathrm{~L}$ baffled flasks (Nalgene) in a shaker incubator at $125 \mathrm{rpm}, 5 \% \mathrm{CO}_{2}$ and $37^{\circ} \mathrm{C}$. Basal medium was Power-CHO-2 (Lonza) supplemented with $8 \mathrm{mM}$ glutamine (Lonza) and gentamicin sulfate $(0.05 \mathrm{mg} / \mathrm{mL})$ (Lonza). The cultures were started in $400 \mathrm{~mL}$ of basal medium at 350,000-400,000 cells $/ \mathrm{mL}$ and were supplemented with feeds: Cell Boost $7 \mathrm{a}$ (Hyclone) at $3 \% \mathrm{v} / \mathrm{v}$ and Cell Boost $7 \mathrm{~b}$ (Hyclone) at $0.3 \% \mathrm{v} / \mathrm{v}$ on days $0,3,4,5,6,8,9$, and 10 . A temperature shift to $34^{\circ} \mathrm{C}$ was done when cell densities reached 5-7 $\times 10^{6}$ cells $/ \mathrm{mL}$. Additional glutamine was added on day 7 , to $2 \mathrm{mM}$. Glucose was added to $4.5 \mathrm{~g} / \mathrm{L}$ when levels dropped below $3.5 \mathrm{~g} / \mathrm{L}$. Cells were harvested on Day 14 or when cell viability fell below $60 \%$.

The secreted proteins were purified by sequential affinity chromatography on nickel and strep-tactin columns (for c-fos/c-jun-based pMHCII), protein A/G columns (for KIH-based pMHCII), and avidin columns (for in vivo-biotinylated empty KIH-based pMHCIIs after protein A/G purification) and used for NP coating or biotinylated in vitro (for peptide-tethered pMHCII) to produce $\mathrm{pMHC}$ tetramers using fluorochrome-conjugated streptavidin.

Molecular modeling. Molecular modeling was done with the DeepView-SwissPdbViewer software ${ }^{34}$. KIH heterodimer modeling was based on the published crystal structure ${ }^{35}$ (Protein Data Bank (PDB) ID: 4NQS). The pMHCII cys-trap model was based on the published crystal structure of $\mathrm{IA}^{\mathrm{g} 7}$ complexed with $\mathrm{GAD}_{207-220}$ (PDB ID: 1ESO) ${ }^{36}$.

SDS-PAGE. The proteins were electrophoresed in 12\% SDS-PAGE gels. To evaluate SDS stability of pMHCII monomers, samples were loaded with $0.83 \%$ SDS and were either boiled $\left(100^{\circ} \mathrm{C}\right.$ for $\left.5 \mathrm{~min}\right)$ or left unboiled. Fully denaturing conditions involved the addition of $20 \mathrm{mM} \beta 2$-ME (Sigma). All uncropped PAGE gel images are shown in Supplementary Fig. 1. 
a

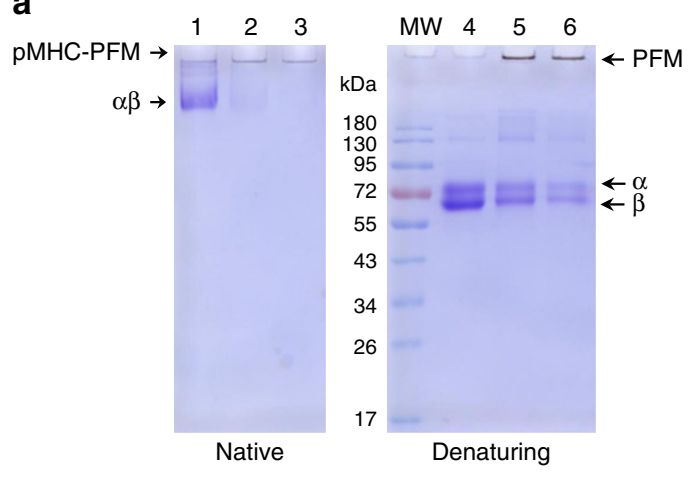

C
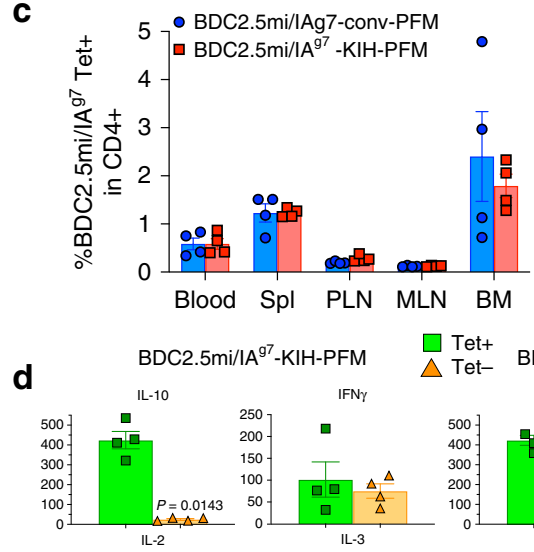

$\square$ Tet+ $\quad$ Tet- $2.5 \mathrm{mi} / \mathrm{IA}^{\mathrm{g} 7}$-conv-PFM
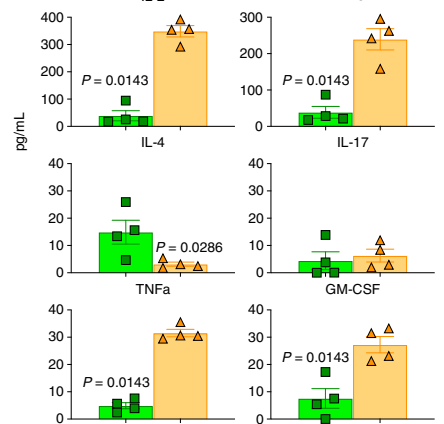
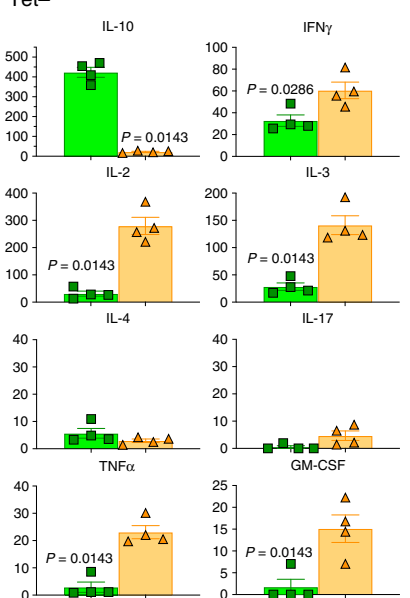

b

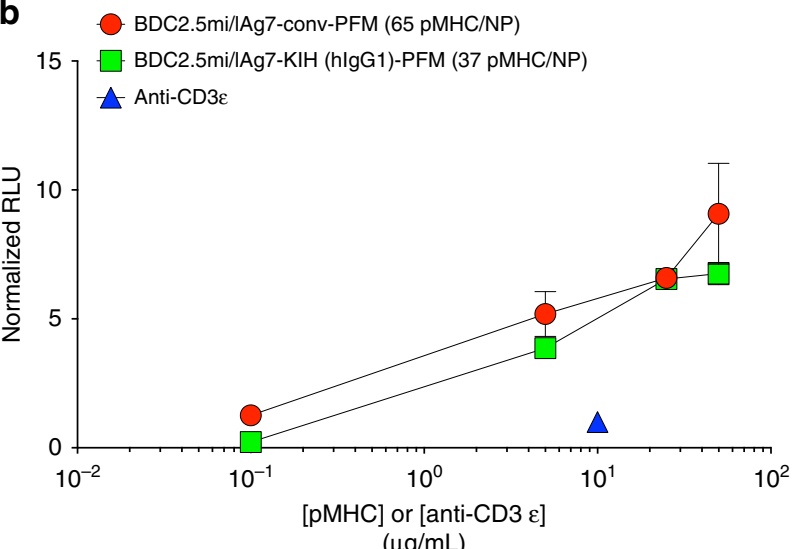

$(\mu \mathrm{g} / \mathrm{mL})$

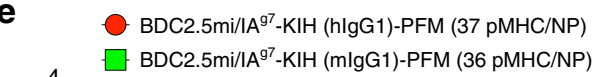

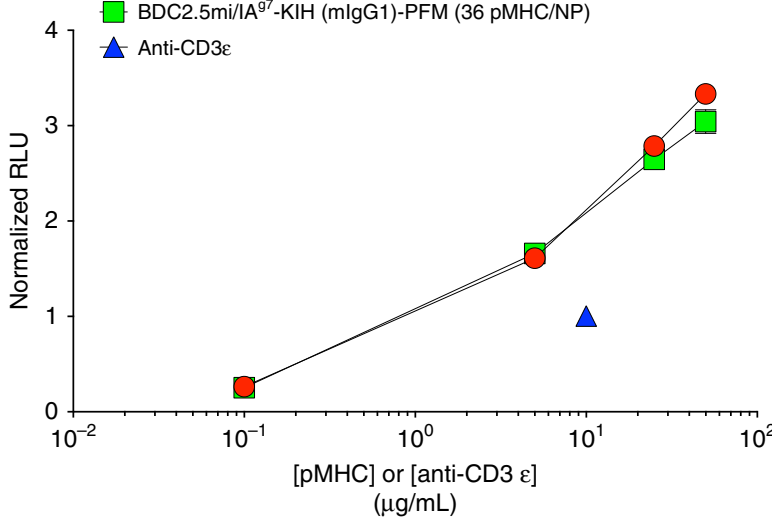

Fig. 7 Biological potency of NPs displaying KIH- vs. c-jun/c-fos-based pMHCII. a Native (left) and denaturing (right) SDS-PAGE for NPs coated with a representative $\mathrm{KIH}$-based $\mathrm{pMHCII}$ molecule. PFM denotes the iron oxide NP. MW: molecular weight markers; $1: 2 \mu \mathrm{g}$ of $\mathrm{KIH}$-based BDC2.5mi/IAg7 monomers; 2: $2.2 \mu \mathrm{L}$ of PFM coated with KIH-based BDC2.5mi/IAg7 monomers; $3: 1.1 \mu \mathrm{L}$ of PFM coated with KIH-based BDC2.5mi/IAg7 monomers; $4: 2 \mu \mathrm{g}$ of $\mathrm{KIH}$-based BDC2.5mi/IAg7 monomers; $5: 2.2 \mu \mathrm{L}$ of PFM coated with KIH-based BDC2.5mi/IAg7 monomers; $6: 1.1 \mu \mathrm{L}$ of PFM coated with KIH-based $\mathrm{BDC} 2.5 \mathrm{mi} / \mathrm{IA}^{\mathrm{g} 7}$ monomers. Electrophoretic behavior of the $\mathrm{KIH}$-based pMHCII-NP compound shown herein is representative of at least 10 different pMHCII-NP preparations made using KIH-based pMHClls. b Luciferase activity induced by NPs coated with c-jun/c-fos- ('conv') or KIH-based BDC2.5mi/ IAg7 monomers (normalized to that induced by soluble anti-CD3e mAb) on Jurkat cells co-expressing mouse CD4, a BDC2.5 mi/IAg7-specific TCR and an NFAT-driven luciferase reporter. Data correspond to mean \pm SEM of triplicates. c Percentages of BDC2.5mi/IAg7 tetramer-positive CD4 ${ }^{+} \mathrm{T}_{\text {-cells in blood, }}$ spleen, pancreatic lymph nodes (PLN), mesenteric lymph nodes (MLN) and bone marrow (BM) from NOD mice treated (twice a week for 5 weeks) with NPs coated with c-jun/c-fos-based ('conv') BDC2.5mi/IAg7 or KIH-based BDC2.5mi/IAg7 monomers (20 $\mu$ g pMHC/dose). Data correspond to average \pm SEM values from 4 mice/group. d Cytokine profile of the tetramer ${ }^{+}$cells isolated from the mice in (c). Tetramer ${ }^{+}$cells were challenged with anti-CD3/ anti-CD28 mAb-coated beads for 3 days and the supernatants assayed for cytokine content using Luminex technology. Data correspond to average \pm SEM values of cells isolated from 4 mice/group. $P$ values were calculated via Mann-Whitney $U$ and are considered significant if $P<0.05$. e Luciferase activity induced by NPs coated with $\mathrm{KIH}$-based BDC2.5 mi/IAg7 pMHCs carrying a mouse or a human Fc-based KIH (normalized to that induced by soluble anti$\mathrm{CD} 3 \varepsilon \mathrm{mAb}$ ) on Jurkat cells co-expressing mouse CD4, a BDC2.5mi/IA $\mathrm{A}^{\mathrm{g}}$-specific TCR and an NFAT-driven luciferase reporter. Data correspond to mean \pm SEM of triplicates. Source data for panels $(\mathbf{b}-\mathbf{e})$ are provided as a Source Data File

In vitro biotinylation of pMHCII monomers. Biotinylation of pMHCII was done by using a biotin-protein ligase kit (BirA enzyme, Avidity). Briefly, $25 \mu \mathrm{M}$ of pMHC was biotinylated with $10 \mu \mathrm{g}$ of BirA enzyme in $50 \mathrm{mM}$ bicine buffer $\mathrm{pH} 8.3$ with 10 $\mathrm{mM}$ magnesium acetate, $10 \mathrm{mM}$ ATP, and $85 \mu \mathrm{M}$ of d-biotin at room temperature overnight. The reaction mixture was dialyzed against $20 \mathrm{mM}$ Tris- $\mathrm{HCl}$ buffer $\mathrm{pH} 8$ and the resulting pMHCII was purified by ion exchange (mono-Q) chromatography. Biotin-conjugated pMHCII fractions were identified via ELISA using horseradish peroxidase-streptavidin (Sigma) and characterized via denaturing SDSPAGE. The biotin-conjugated pMHCII fractions were pooled, buffer exchanged into PBS by spin ultrafiltration (Millipore, MW cut-off $30 \mathrm{KDa}$ ) and stored at $-80^{\circ} \mathrm{C}$. 
a

- BDC2.5mi//Ag7-Conv-PFM (65 pMHC/NP)

$\square$ BDC2.5mi//Ag7-KIH(hlgG1)-PGM (37 pMHC/NP) \pm Anti-CD3

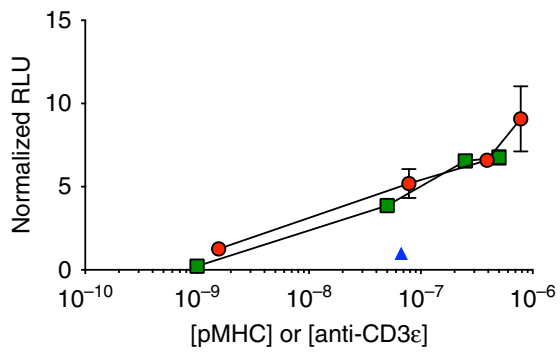

[M]

C

C $\mathrm{BDC} 2.5 \mathrm{mi} / \mathrm{Ag} 7-\mathrm{KIH}(\mathrm{hlgG} 1)-\mathrm{PFM}(37 \mathrm{pMHC} / \mathrm{NP})$

$\triangle$ Anti-CD3

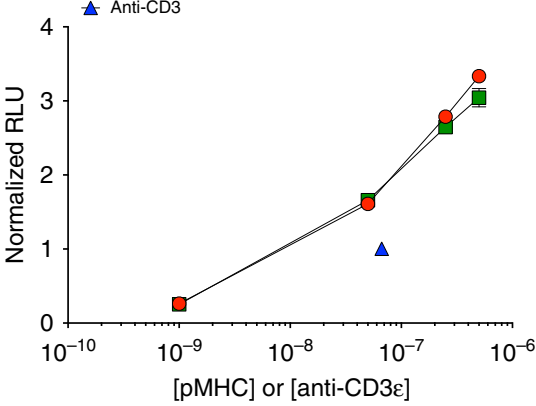

[M]

e IGRP13-25/DR3-conv-CT-PFM (63 pMHC/NP)

^ IGRP13-25/Dr3-KIH-Non-CT-PFM (46 pMHC/NP)

$\nabla$ IGRP13-25/DR3-KIH-Non-CT-PFM (29 pMHC/NP)

IGRP13-25/DR3-KIH-Non-CT-PFM (27 pMHC/NP)

4 Anti-CD3e

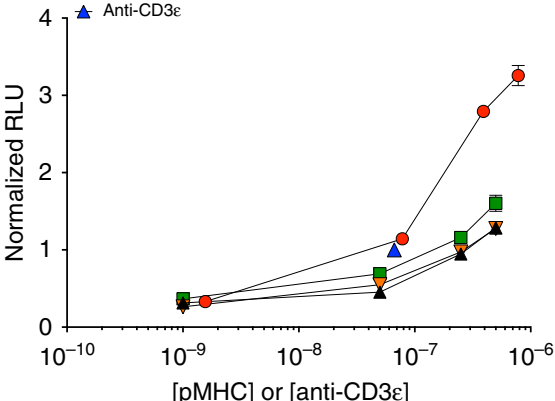

[M]

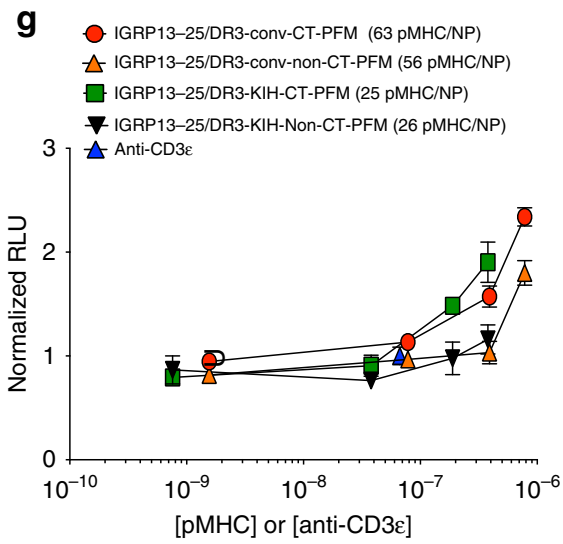

[M]

pMHC tetramers. Phycoerythrin (PE)-conjugated tetramers were prepared using biotinylated pMHCII monomers and used to stain peripheral T-cells or TCRtransfected Jurkat cell lines ${ }^{37,38}$. "Empty" MHCII complexes were biotinylated in BirA-transgenic CHO-S cells and purified on avidin columns. Briefly, CHO-S cells were transduced with lentiviruses encoding each of the two chains of the KIH constructs as described above. BirA-ER enzyme (Addgene) was cloned into another lentiviral plasmid carrying human $\mathrm{CD} 4$ as a reporter gene and used to transduce b

BDC2.5mi//Ag7-Conv-PFM (65 pMHC/NP)

$\square \mathrm{BDC} 2.5 \mathrm{mi} / / \mathrm{Ag} 7-\mathrm{KIH}$ (hlgG1)-PGM (37 pMHC/NP)

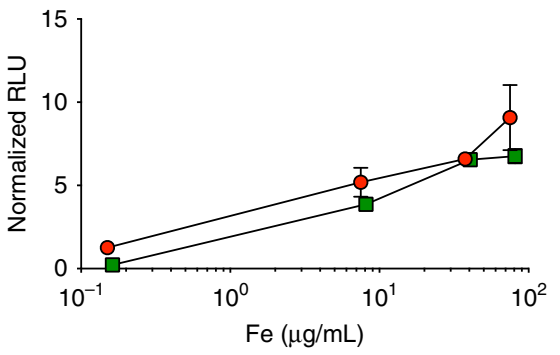

d

BDC2.5mi//Ag7-KIH(hlgG1)-PFM (37 pMHC/NP)

BDC2.5mi//Ag7-KIH(mlgG1)-PFM (36 pMHC/NP)

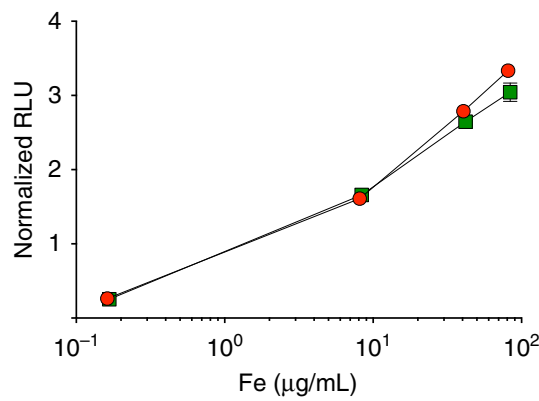

f IGRP13-25/DR3-conv-CT-PFM (63 pMHC/NP)

^ IGRP13-25/DR3-KIH-Non-CT-PFM (46 pMHC/NP)

$\forall$ IGRP13-25/DR3-KIH-Non-CT-PFM (29 pMHC/NP)

$\rightarrow$ IGRP13-25/DR3-KIH-Non-CT-PFM (27 pMHC/NP)

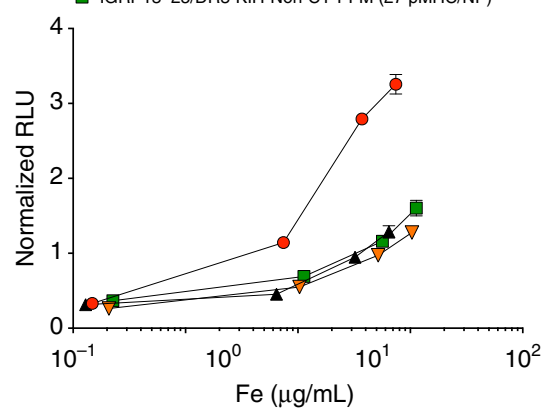

h

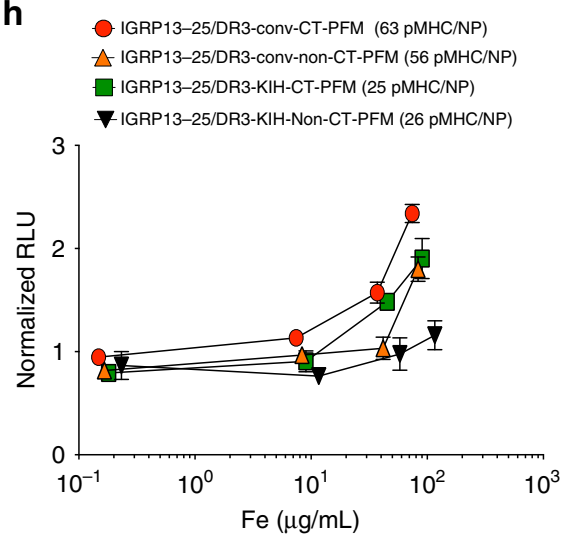

CHO-S cell lines expressing the different KIH-based MHCIIs. Cells were FACSsorted based on positivity for GFP, CFP, and human CD4 using a Becton Dickinson FACsAriaII sorter. Cell lines were expanded and grown to a density of $10-15^{7}$ cells $/ \mathrm{mL}$ during 14 days in the presence of $2 \mu \mathrm{g} / \mathrm{mL}$ of biotin (ThermoFisher). Biotinylated soluble MHCII molecules were purified from culture supernatants by protein $\mathrm{G}$ column chromatography using an ÄKTA protein purification system (GE). PBS or $20 \mathrm{mM}$ Trizma buffer exchange was done using a size 
Fig. 8 TCR signaling potency of pMHC-NPs as a function of pMHCII molarity or NP number. $\mathbf{a}, \mathbf{b}$ Luciferase activity induced by NPs coated with c-jun/cfos- ('conv') or $\mathrm{KIH}$-based BDC2.5mi//Ag7 monomers (normalized to that induced by soluble anti-CD3ع mAb) on Jurkat cells co-expressing mouse CD4, a BDC2.5mi/IAg7-specific TCR and an NFAT-driven luciferase reporter. Data correspond to main Fig. 7b but normalized by molar concentration of pMHCII or NP number. c, d Luciferase activity induced by NPs coated with KIH-based BDC2.5mi/IAg7 pMHClls carrying a mouse or a human Fc-based KIH

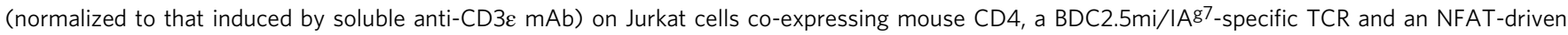
luciferase reporter. Data correspond to main Fig. 7e but normalized by molar concentration of pMHCII or NP number. e, $\mathbf{f}$ Luciferase activity induced by

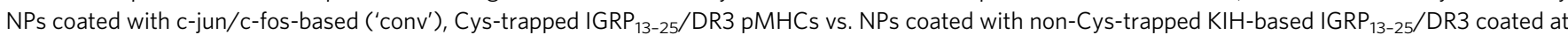

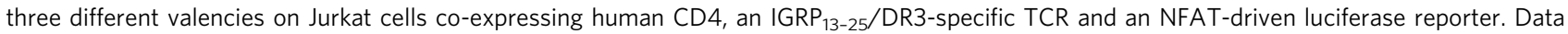
correspond to main Fig. 9d but normalized by molar concentration of pMHCII or NP number. $\mathbf{g}$, $\mathbf{h}$ Luciferase activity induced by NPs coated with c-jun/cfos-based/Cys-trapped or KIH-based/Cys-trapped IGRP ${ }_{13-25}$ /DR3 monomers vs. their non-Cys-trapped counterparts on Jurkat cells co-expressing human CD4, an IGRP 13-25/DR3-specific TCR and an NFAT-driven luciferase reporter. Data correspond to main Fig. 9e but normalized by molar concentration of pMHCII or NP number. Data correspond to mean \pm SEM of triplicates. Source data are provided as a Source Data File

exclusion column (GE). A second purification using avidin column kit was done in order to purify in vivo-biotinylated proteins (ThermoFisher).

The biotinylated molecules were then loaded with peptide by incubation with a 10-fold molar excess of PDC-E2 $2_{122-135}$ (Tebu-bio), PDC-E2 $249-262$ (Tebu-bio), and IGRP $_{13-25}$ (Genscript) in $100 \mathrm{mM} \mathrm{NaPO}_{4} \mathrm{pH} 6.0,0.2 \%$ n-octyl-d-glucopyranoside (Sigma-Aldrich) and $1 \mathrm{mg} / \mathrm{ml} \mathrm{Pefabloc}{ }^{\oplus}$ (Sigma-Aldrich) for $72 \mathrm{~h}$ at $37^{\circ} \mathrm{C}$. The peptide-loaded MHCII molecules were then incubated with PE-streptavidin (ThermoFisher) at a 5:1 molar ratio overnight at room temperature to generate tetrameric pMHCII complexes.

Flow cytometry. To stain mononuclear cell suspensions from NOD mice, peripheral blood, splenocytes, lymph node and bone marrow cell suspensions were incubated with avidin for $15 \mathrm{~min}$ at room temperature and stained with tetramer $(10-33 \mu \mathrm{g} / \mathrm{mL}$, see below) in FACS buffer (0.05\% sodium azide and 1\% FBS in PBS) for $30 \mathrm{~min}$ at $4{ }^{\circ} \mathrm{C}$, washed, and incubated with FITC-conjugated anti-CD4 $(5 \mu \mathrm{g} /$ $\mathrm{mL})$ and PerCP-conjugated anti-B220 $(2 \mu \mathrm{g} / \mathrm{mL}$; as a 'dump' channel) for $30 \mathrm{~min}$ at $4{ }^{\circ} \mathrm{C}$, in the presence of an anti-CD16/CD $32 \mathrm{mAb}(2.4 \mathrm{G} 2$; BD Pharmingen) to block FcRs. Cells were washed, fixed in 1\% paraformaldehyde (PFA) in PBS and analyzed with FACScan, FACSaria, BD LSRII, FACSCanto or Fortessa flow cytometers. Analysis was done using FlowJo software.

TCR-transduced Jurkat cell lines were stained with $10 \mu \mathrm{g} / \mathrm{ml}$ of c-jun/c-fosbased pMHCII tetramer or $33 \mu \mathrm{g} / \mathrm{ml} \mathrm{KIH-based} \mathrm{tetramer} \mathrm{in} 50 \mu \mathrm{l}$ of PBS for $1 \mathrm{~h}$ at $37^{\circ} \mathrm{C}$. Propidium iodide (Sigma, St. Louis, Missouri, USA) was added $5 \mathrm{~min}$ before analysis to discriminate live from dead cells.

For experiments using PBMCs spiked with clonal cells, experimental samples were created by mixing clonal T-cells $\left(10^{4}\right)$ with PBMCs $\left(10^{6}\right)$. The PBMCs were HLA-matched for the restricting HLA of the T-cell clone used. Some samples were treated prior to tetramer staining with the protein kinase inhibitor (PKI) Dasatinib (Axon Medchem) at $50 \mathrm{nM}$ for $30 \mathrm{~min}$ at $37^{\circ} \mathrm{C}$. Tetramer staining was performed with $33 \mu \mathrm{g} / \mathrm{ml}$ of peptide-loaded KIH-based pMHC tetramers in $50 \mu \mathrm{l}$ of PBS for $1 \mathrm{~h}$ at $37^{\circ} \mathrm{C}$. All samples were subsequently stained with anti-CD4 (aCD4) (OKT4; BioLegend), to gate on $\mathrm{CD} 4^{\text {hi }}$ cells and, in some cases, with anti-human Fc-PE (Jackson ImmunoResearch) for $20 \mathrm{~min}$ at $37^{\circ} \mathrm{C}$. Propidium iodide (Sigma) was added $5 \mathrm{~min}$ before analysis to discriminate live from dead cells. FACS gating strategies are provided in Supplementary Fig. 2.

NP synthesis. Maleimide-functionalized, pegylated iron oxide NPs (PFM series) were produced in a single-step thermal decomposition in the absence of surfactants ${ }^{4}$. Briefly, $3 \mathrm{~g}$ Maleimide-PEG ( $2 \mathrm{kDa}$ MW, Jenkem Tech, USA) were melted in a $50 \mathrm{~mL}$ round bottom flask at $100^{\circ} \mathrm{C}$ and then mixed with $7 \mathrm{~mL}$ of benzyl ether and $2 \mathrm{mmol} \mathrm{Fe}(\mathrm{acac})_{3}$. The reaction was stirred for $1 \mathrm{~h}$ and heated to $260^{\circ} \mathrm{C}$ with reflux for $2 \mathrm{~h}$. The mixture was cooled to room temperature and mixed with $30 \mathrm{~mL}$ water. Insoluble materials were removed by centrifugation at $2000 \times g$ for $30 \mathrm{~min}$. The NPs were purified using magnetic (MACS) columns (Miltenyi Biotec) and stored in water at room temperature or $4{ }^{\circ} \mathrm{C}$. The concentration of iron was determined spectrophotometrically at $410 \mathrm{~nm}$ in $2 \mathrm{~N}$ hydrochloric acid ( $\mathrm{HCl})$.

pMHCII conjugation to NPs. pMHCII conjugation to maleimide-functionalized NPs (PF-M) was done via the free C-terminal Cys engineered into the MHCa chain/Knob. Briefly, pMHCs were mixed with NPs in $40 \mathrm{mM}$ phosphate buffer, $\mathrm{pH}$ 6.0 , containing $2 \mathrm{mM}$ ethylenediaminetetraacetic acid (EDTA), $150 \mathrm{mM} \mathrm{NaCl}$, and incubated overnight at room temperature. pMHC-conjugated NPs were purified by magnetic separation and concentrated by ultrafiltration through Amicon Ultra-15 (100-300 kDa cut-off) and stored in PBS.

NP characterization. The size and dispersity of unconjugated and pMHCIIconjugated NPs were assessed via transmission electron microscopy (TEM, Hitachi H7650) and dynamic light scattering (DLS, Zetasizer, Malvern). Pegylated and pMHC-NPs were analyzed via $0.8 \%$ agarose gel electrophoresis, native and denaturing $10 \%$ SDS-PAGE. To quantify pMHC valency, we measured the pMHC concentration of the pMHC-NP preps using the Bradford assay (Thermo Scientific).
Reactivity of hMHClls to conformation epitope-specific mAbs. The KIH-based pMHC monomers were diluted to an identical concentration $(200 \mathrm{ng} / \mathrm{mL})$ and serially diluted. A sandwich ELISA assay was used to capture and quantify the pMHCs. Briefly, plates were coated with goat anti-human IgG (Jackson ImmunoResearch) (working concentration $24 \mu \mathrm{g} / \mathrm{mL}$ ) as a capture antibody. The capture antibody $(100 \mu \mathrm{L} /$ well $)$ was incubated in a 96-well flat bottom Immuno plate (Thermo Scientific) overnight at room temperature. The plates were blocked using PBS containing $1 \%$ BSA and $0.05 \%$ sodium azide for $1 \mathrm{~h}$. The plates were then washed four times with PBS containing $0.5 \%$ Triton X-100, $200 \mu \mathrm{L} /$ well (washing buffer). The serially diluted pMHC-human KIH fusion protein solution $(100 \mu \mathrm{L} /$ well) was added to the wells and incubated for $2 \mathrm{~h}$ at room temperature. The plates were washed four times. The captured pMHCIIs were then detected using biotinylated anti-human HLA-DR mAb (clone L243, from Biolegend; $0.4 \mu \mathrm{g} / \mathrm{well}$, $100 \mu \mathrm{L} /$ well). The plates were incubated with the capture antibody for $2 \mathrm{~h}$ at room temperature, washed four times and then incubated with ExtrAvidin Peroxidase Conjugate (Sigma-Aldrich; 1:2000 dilution in PBS, $100 \mu \mathrm{L} /$ well) for $30 \mathrm{~min}$ at room temperature. The plates were washed again, and incubated with 3,3',5,5'-Tetramethylbenzidine (TMB, Sigma-Aldrich; $100 \mu \mathrm{L} /$ well) for $5 \mathrm{~min}$. The color reaction was stopped by adding $50 \mu \mathrm{L}$ of $2 \mathrm{~N} \mathrm{H}_{2} \mathrm{SO}_{4}$. The absorbance of the reaction was measured at $450 \mathrm{~nm}$ and $570 \mathrm{~nm}$ wavelengths using a plate reader (SpectraMax i3x, Molecular Devices).

TCR signaling in TCR/CD4-transfected Jurkat cells. The TCR $\alpha$ and TCR $\beta$ cDNAs encoding the BDC2.5-TCR were generated from BDC2.5-CD4 ${ }^{+}$T-cellderived mRNA using the $5^{\prime}$ RACE System for Rapid Amplification of cDNA Ends, version 2.0 kit (Thermo-Fisher Scientific), and subcloned as a P2A-tethered single open-reading frame into a retroviral vector upstream of an IRES-eGFP cassette. The TCR cDNAs encoding human IGRP ${ }_{13-25} / \mathrm{DR} 3-$, PDC-E2 ${ }_{122-135} / \mathrm{DRB} 4{ }^{*} 0101 /$ DRA1*-0101-, and PDC-E2 $249-262 / D R B 4^{*} 0101 / D R A 1{ }^{*}-0101$-specific TCRs were cloned from human T-cell clones generated from T1D or primary biliary cholangitis (PBC) patients ${ }^{4}$. Briefly, PBMCs, obtained from PBC patients recruited under informed consent approved by the Institutional Review Board at Hospital Clinic, were isolated from heparinized blood by gradient centrifugation and resuspended at $5 \times 10^{6} / \mathrm{mL}$ in RPMI- 1640 media supplemented with $10 \%$ human $\mathrm{AB}$ serum. The cells were cultured in 24 -well plates in the presence of $10 \mu \mathrm{g} / \mathrm{mL}$ peptide. After 7-10 days, cells were washed, and cultured for 5 days in wells coated, at high density, with avidin and biotinylated pMHCII monomer. Finally, cells were cultured for 5 additional days in the presence of $1 \mu \mathrm{g} / \mathrm{mL}$ of soluble anti-hCD28 $\mathrm{mAb}$ (BD Pharmingen) followed by interleukin-2 (R\&D). Tetramer ${ }^{+} \mathrm{CD}^{+}{ }^{+} \mathrm{T}$-cells were single-cell sorted into 96-well plates using a FACSAriaII sorter (Becton Dickinson) and used for TCR sequencing 4 .

The human $\mathrm{CD}^{+} / \mathrm{TCR} \beta^{-}$JurMA (Jurkat) reporter cell line (expressing NFATdriven luciferase) was transduced with retroviruses encoding mouse or human $\mathrm{CD} 4$ and mouse or human TCR $\beta \beta$, respectively. eGFP and mouse or human CD4 double-positive cells were sorted by flow cytometry and stained with PE-labeled pMHCII tetramers to confirm specificity.

To measure NFAT-driven expression of luciferase, wild-type and BDC2.5/ $\mathrm{mCD}^{+}$or $\mathrm{IGRP}_{13-35} / \mathrm{DR} 3-\mathrm{TCR} / \mathrm{hCD} 4^{+}$Jurkat cells were plated at 500,000 cells/ $\mathrm{mL}$ in $200 \mu \mathrm{l}$ of DMEM (Sigma-Aldrich) supplemented with 10\% FBS (SigmaAldrich) in the presence or absence of $10 \mu \mathrm{g} / \mathrm{mL}$ of anti-hCD3 $\varepsilon \mathrm{mAb}$ (OKT3, BD Biosciences) or various concentrations of pMHC-coated PFM for $12 \mathrm{~h}$. Cells were washed three times with PBS and $10^{5}$ cells lysed in $20 \mu \mathrm{l}$ Cell Culture Lysis Reagent (Promega) and incubated with $100 \mu \mathrm{l}$ of Luciferase Assay Reagent (Promega) in opaque white plates (Greiner Bio One International GmbH) using a Veritas ${ }^{\text {mo }}$ Microplate Luminometer (Promega) with injectors. Luciferase activity was expressed as relative luminescence units (RLUs), normalized to the luciferase activity of anti-CD $3 \varepsilon \mathrm{mAb}$-challenged cells.

pMHCII-NP therapy of NOD mice. Cohorts of 10-week-old female NOD mice were injected i.v. with pMHCII-coated NPs in PBS ( $20 \mu \mathrm{g}$ pMHC/dose) twice a week for 5 weeks. Increases in the size of tetramer ${ }^{+} \mathrm{CD} 4^{+} \mathrm{T}$-cell pools in blood, 
a

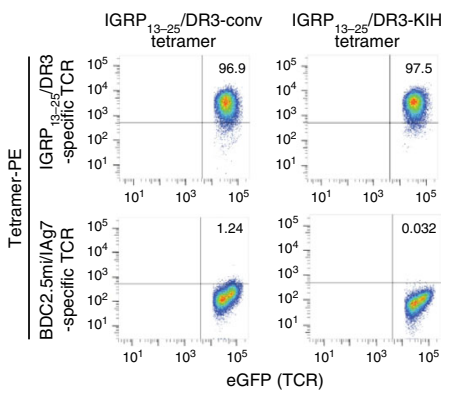

C

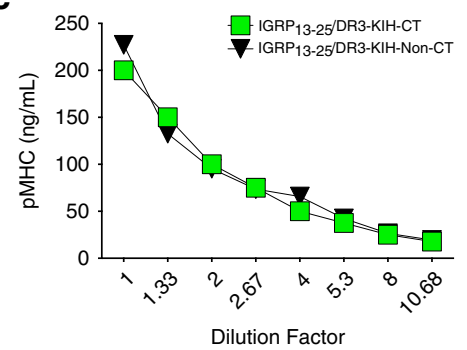

e

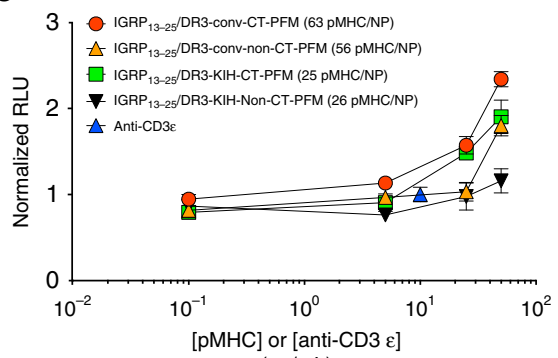
$(\mu \mathrm{g} / \mathrm{mL})$
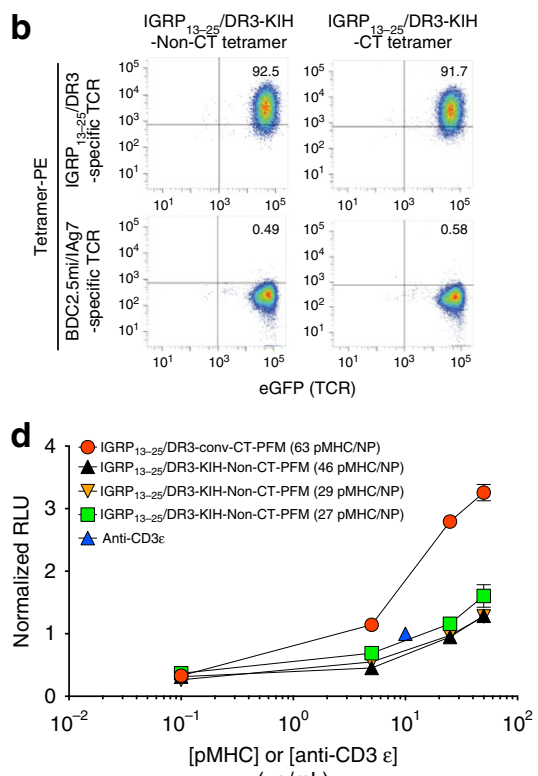

$(\mu \mathrm{g} / \mathrm{mL})$
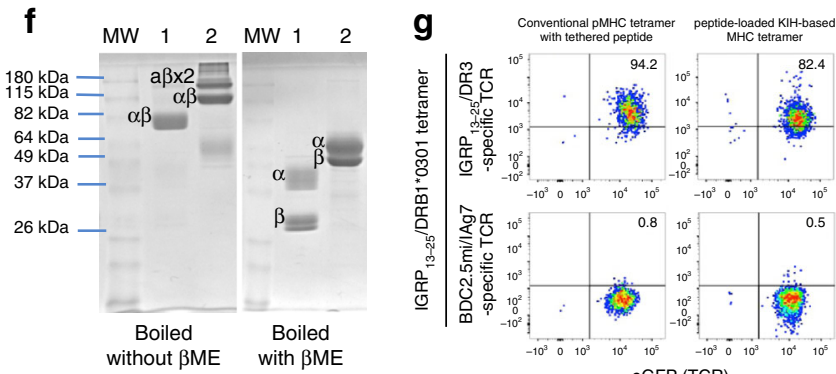

eGFP (TCR)
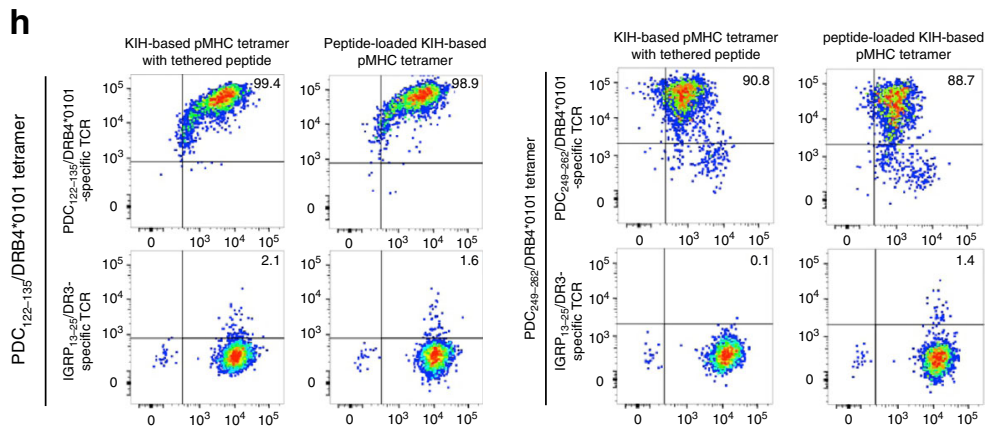

i

thout dasatinib

eGFP (TCR)

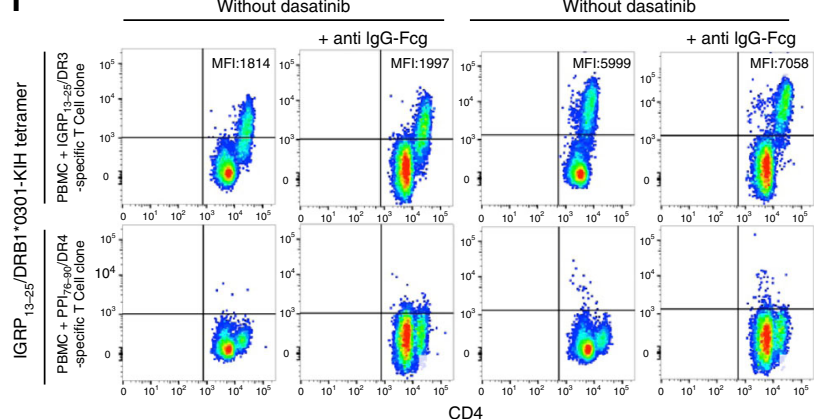

spleen, lymph nodes and/or marrow, as well as their phenotypic properties, were assessed by flow cytometry as described ${ }^{2}$.

Cytokine secretion assay. $\mathrm{CD} 4^{+} \mathrm{T}$-cells from pMHC-NP-treated mice were enriched from spleen cell suspensions using a BD Imag enrichment kit, stained with pMHCII tetramers as described above and sorted into tetramer ${ }^{+}$and tetramer ${ }^{-}$subsets by flow cytometry. FACS-sorted cells $\left(2-3 \times 10^{4}\right)$ were stimulated with anti-CD3/anti-CD28 mAb-coated beads for $48 \mathrm{~h}$ and the supernatants collected $48 \mathrm{~h}$ later for measurement of cytokines via Luminex.

Statistical analyses. Quantitative data were compared by Mann-Whitney U. Statistical significance was assumed at $P<0.05$. 
Fig. 9 The KIH Fc affords increased biological potency and stabilizes "empty" MHCIl. a Representative pMHCII tetramer/eGFP (TCR) FACS dot plots for Jurkat cells expressing hCD4 and an IGRP $13-25 / D R 3$-specific TCR or mCD4 and a BDC2.5mi/IA ${ }^{g 7}$-specific TCR (negative control). b Representative pMHCII tetramer/eGFP (TCR) dot plots for the Jurkat cells in A, but stained with KIH-based tetramers lacking (left) or carrying a CT (right). c Introduction of a $\mathrm{CT}$ into $\mathrm{KIH}$-based human pMHCII does not alter their reactivity with a MHCIl conformational epitope-specific mAb, as measured by ELISA. Data correspond to mean \pm SEM of triplicates. $\mathbf{d}$ Luciferase activity induced by NPs coated with c-jun/c-fos-based ('conv'), CT IGRP $13-25 / D R 3$ pMHCs vs. NPs

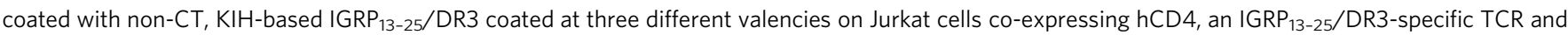
NFAT-luciferase. Data correspond to mean \pm SEM of triplicates. e Luciferase activity induced by NPs coated with c-jun/c-fos-based/CT ('conv') or KIH-

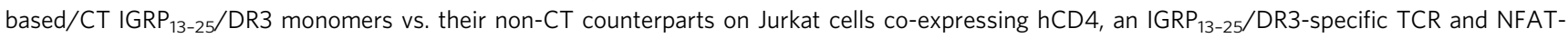
luciferase. Data correspond to mean \pm SEM of triplicates. f SDS-PAGE of CT leucine-zippered (1) or KIH-based (2) Gliadin $62-72 / D Q B 1^{\star} 0201 / D Q A 1^{\star} 0501$ monomers. $\beta M E$, beta-mercaptoethanol. $\mathbf{g}$ Representative $\mathrm{pMHCll}$ tetramer/CD4 dot plots for Jurkat cells expressing hCD4 and an IGRP $13-25 / \mathrm{DR}$-specific

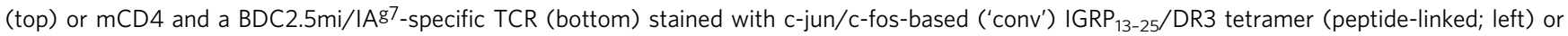
tetramers generated using peptide-loaded empty DR3-KIH monomers (right). h Representative pMHCII tetramer/eGFP (TCR) plots for Jurkat cells

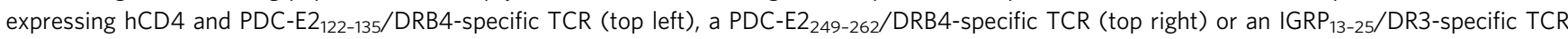
(bottom; negative control). i Signal amplification of $\mathrm{KIH}$-based tetramer binding using anti-hFc. Human PBMCs (106) were spiked with cells from a

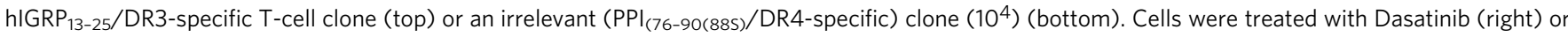
left untreated (left) and then stained with PE-labeled tetramers and PE-labeled anti-lgG. Values on the plots correspond to the geometric mean fluorescence intensity for pMHC tetramer staining. The staining patterns shown in (a), (b), ( $\mathbf{g})$, and (h) are representative of at least two independent experiments. Source data for panels (c-e) are provided as a Source Data File

Reporting summary. Further information on research design is available in the Nature Research Reporting Summary linked to this article.

\section{Data availability}

The source data underlying Figs. $7 \mathrm{~b}-\mathrm{e}, 8 \mathrm{a}-\mathrm{d}, 9 \mathrm{c}-\mathrm{e}$ and $10 \mathrm{a}-\mathrm{d}$ are provided as a Source Data File. Uncropped gel images and FACS gating strategies are provided in the Supplementary Information.

Received: 12 July 2019; Accepted: 4 October 2019;

Published online: 29 October 2019

\section{References}

1. Altman, J. D. et al. Phenotypic analysis of antigen-specific T lymphocytes. Science 274, 94-96 (1996).

2. Clemente-Casares, X. et al. Expanding antigen-specific regulatory networks to treat autoimmunity. Nature 530, 434-440 (2016).

3. Tsai, S. et al. Reversal of autoimmunity by boosting memory-like autoregulatory T cells. Immunity 32, 568-580 (2010).

4. Singha, S. et al. Peptide-MHC-based nanomedicines for autoimmunity function as T-cell receptor microclustering devices. Nat. Nanotechnol. 12 701-710 (2017).

5. Umeshappa, C. S. et al. Supression of a broad spectrum of liver autoimmune pathologies by single peptide-MHC-based nanomedicines. Nat. Commun. 10, 1-17 (2019).

6. Cosson, P. \& Bonifacino, J. S. Role of transmembrane domain interactions in the assembly of class II MHC molecules. Science 258, 659-662 (1992).

7. Vollers, S. S. \& Stern, L. J. Class II major histocompatibility complex tetramer staining: progress, problems, and prospects. Immunology 123, 305-313 (2008).

8. Merchant, A. M. et al. An efficient route to human bispecific IgG. Nat. Biotechnol. 16, 677-681 (1998).

9. Carter, P. Bispecific human IgG by design. J. Immunol. Methods 248, 7-15 (2001).

10. Sadegh-Nasseri, S. \& Germain, R. N. A role for peptide in determining MHC class II structure. Nature 353, 167-170 (1991).

11. Sadegh-Nasseri, S. \& Germain, R. N. How MHC class II molecules work: peptide-dependent completion of protein folding. Immunol. Today 13, 43-46 (1992).

12. Verreck, F. A. et al. The generation of SDS-stable HLA DR dimers is independent of efficient peptide binding. Int. Immunol. 8, 397-404 (1996).

13. Nelson, C. A., Petzold, S. J. \& Unanue, E. R. Identification of two distinct properties of class II major histocompatibility complex-associated peptides. Proc. Natl Acad. Sci. USA 90, 1227-1231 (1993).

14. Stadinski, B. D. et al. Diabetogenic T cells recognize insulin bound to IAg7 in an unexpected, weakly binding register. Proc. Natl Acad. Sci. USA 107, 10978-10983 (2010)

15. Yang, J. et al. Autoreactive T cells specific for insulin B:11-23 recognize a lowaffinity peptide register in human subjects with autoimmune diabetes. Proc. Natl Acad. Sci. USA 111, 14840-14845 (2014).

16. Casares, $\mathrm{S}$. et al. Down-regulation of diabetogenic CD4+ T cells by a soluble dimeric peptide-MHC class II chimera. Nat. Immunol. 3, 383-391 (2002).
17. Preda, I. et al. Soluble, dimeric HLA DR4-peptide chimeras: an approach for detection and immunoregulation of human type-1 diabetes. Eur. J. Immunol. 35, 2762-2775 (2005).

18. Moro, M. et al. Generation of functional HLA-DR ${ }^{\star} 1101$ tetramers receptive for loading with pathogen- or tumour-derived synthetic peptides. $B M C$ Immunol. 6, 24 (2005).

19. Goldberg, B. \& Bona, C. Dimeric MHC-peptides inserted into an immunoglobulin scaffold as new immunotherapeutic agents. J. Cell. Mol. Med. 15, 1822-1832 (2011).

20. Gorga, J. C., Horejsi, V., Johnson, D. R., Raghupathy, R. \& Strominger, J. L. Purification and characterization of class II histocompatibility antigens from a homozygous human B cell line. J. Biol. Chem. 262, 16087-16094 (1987).

21. Stockel, J. et al. Refolding of human class II major histocompatibility complex molecules isolated from Escherichia coli. Assembly of peptide-free heterodimers and increased refolding-yield in the presence of antigenic peptide. J. Biol. Chem. 269, 29571-29578 (1994).

22. Quarsten, H. et al. Staining of celiac disease-relevant $\mathrm{T}$ cells by peptide-DQ2 multimers. J. Immunol. 167, 4861-4868 (2001).

23. James, E. A. et al. Tetramer-guided epitope mapping reveals broad, individualized repertoires of tetanus toxin-specific CD4 $+\mathrm{T}$ cells and suggests HLA-based differences in epitope recognition. Int. Immunol. 19, 1291-1301 (2007).

24. Novak, E. J. et al. Tetramer-guided epitope mapping: rapid identification and characterization of immunodominant $\mathrm{CD} 4+\mathrm{T}$ cell epitopes from complex antigens. J. Immunol. 166, 6665-6670 (2001).

25. Novak, E. J., Liu, A. W., Nepom, G. T. \& Kwok, W. W. MHC class II tetramers identify peptide-specific human $\mathrm{CD} 4(+) \mathrm{T}$ cells proliferating in response to influenza A antigen. J. Clin. Invest. 104, R63-R67 (1999).

26. Wallny, H. J., Sollami, G. \& Karjalainen, K. Soluble mouse major histocompatibility complex class II molecules produced in Drosophila cells. Eur. J. Immunol. 25, 1262-1266 (1995).

27. Yang, J., Jaramillo, A., Shi, R., Kwok, W. W. \& Mohanakumar, T. In vivo biotinylation of the major histocompatibility complex (MHC) class II/peptide complex by coexpression of BirA enzyme for the generation of MHC class II/ tetramers. Hum. Immunol. 65, 692-699 (2004).

28. Dolton, G. et al. More tricks with tetramers: a practical guide to staining T cells with peptide-MHC multimers. Immunology 146, 11-22 (2015).

29. Lissina, A. et al. Protein kinase inhibitors substantially improve the physical detection of T-cells with peptide-MHC tetramers. J. Immunol. Methods 340, 11-24 (2009).

30. Batard, P. et al. Dextramers: new generation of fluorescent MHC class I/ peptide multimers for visualization of antigen-specific CD8+ T cells. J. Immunol. Methods 310, 136-148 (2006).

31. Crawford, F. et al. Use of baculovirus MHC/peptide display libraries to characterize T-cell receptor ligands. Immunol. Rev. 210, 156-170 (2006).

32. Katz, J. D., Wang, B., Haskins, K., Benoist, C. \& Mathis, D. Following a diabetogenic $\mathrm{T}$ cell from genesis through pathogenesis. Cell 74, 1089-1100 (1993).

33. Holst, J. et al. Generation of T-cell receptor retrogenic mice. Nat. Protoc. 1, 406-417 (2006)

34. Guex, N. \& Peitsch, M. C. SWISS-MODEL and the Swiss-PdbViewer: an environment for comparative protein modeling. Electrophoresis 18, 2714-2723 (1997). 
35. Elliott, J. M. et al. Antiparallel conformation of knob and hole aglycosylated half-antibody homodimers is mediated by a $\mathrm{CH} 2-\mathrm{CH} 3$ hydrophobic interaction. J. Mol. Biol. 426, 1947-1957 (2014).

36. Corper, A. L. et al. A structural framework for deciphering the link between I$\mathrm{Ag} 7$ and autoimmune diabetes. Science 288, 505-511 (2000).

37. Amrani, A. et al. Progression of autoimmune diabetes driven by avidity maturation of a T-cell population. Nature 406, 739-742 (2000).

38. Stratmann, T. et al. The I-Ag7 MHC class II molecule linked to murine diabetes is a promiscuous peptide binder. J. Immunol. 165, 3214-3225 (2000).

\section{Acknowledgements}

We thank S. Thiessen, J. Erickson, and G. Mendizabal for technical contributions and animal care, and Y. Liu, L. Kennedy, and K. Poonam for flow cytometry. This work was funded by Diabetes Canada, the Canadian Institutes of Health Research (CIHR), Ministerio de Economia y Competitividad of Spain (MINECO) and Generalitat de Catalunya (SGR and CERCA Programmes), and the Novartis Institute for Biomedical Research (NIBR). P. Serra was an investigator of the Ramon y Cajal re-integration programme and was supported by a JDRF Career Development Award. D.P., P. Solè, and J.G. were supported by studentships from MINECO. J.B. was supported by Rio Hortega (MINECO) and European Foundation for the Study of Diabetes (EFSD-Lilly) fellowships. P.S. was a Scientist of the AI-HS and a scholar of the ISCIII. The JMDRC is supported by Diabetes Canada.

\section{Author contributions}

N.G., D.P., P. Solé, J.B., M.T., and J.G. produced lentiviruses, cell lines and pMHCs. P. Solè and J.G. generated the IGRP ${ }_{13-25} / \mathrm{DR}^{2}$ - and $\mathrm{BDC}_{2} .5 \mathrm{mi} / \mathrm{IA}^{\mathrm{g} 7}-$, or $\mathrm{PDC}_{122-135} /$ DRB4 ${ }^{\star} 0101-$ and $\mathrm{PDC}_{249-262} / \mathrm{DRB} 4^{\star} 0101$-specific Jurkat TCR transfectants, respectively. C.F. coordinated pMHC expression, purified pMHCs and ran SDS-PAGE. M.O. contributed to quality control. N.G. and S.S. generated biotinylated pMHC monomers and tetramers and did tetramer staining for Figs. 9B-D (N.G.) and 5C, 6E, 7A and 9A, B (S. S.). S.S. generated pMHC-NPs. J.Y. produced the data for Figs. 7C and D. Y.Y. generated data for Fig. 8C. K.E. produced Figs. 7B, 7E, 9D, 9E, 8, and 10. P. Serra engineered the various constructs. P. Santamaria designed the study and coordinated its execution and wrote the paper with P. Serra.

\section{Competing interests}

A patent application on the KIH-based heterodimerization strategy has been filed in the US patent office (Inventor: P. Santamaria; Applicant: University Technologies International, University of Calgary; Application Number: 15/807415; Status: pending). P.

Santamaria is Scientific Founder of Parvus Therapeutics, Inc. and declares a financial interest in the company. The other authors declare no competing interests.

\section{Additional information}

Supplementary information is available for this paper at https://doi.org/10.1038/s41467 019-12902-2.

Correspondence and requests for materials should be addressed to P.S. or P.S.

Peer review information Nature Communications thanks Scheherazade Sadegh-Naseri and other, anonymous, reviewer for their contributions to the peer review of this.

Reprints and permission information is available at http://www.nature.com/reprints

Publisher's note Springer Nature remains neutral with regard to jurisdictional claims in published maps and institutional affiliations.

cc) (i) Open Access This article is licensed under a Creative Commons Attribution 4.0 International License, which permits use, sharing, adaptation, distribution and reproduction in any medium or format, as long as you give appropriate credit to the original author(s) and the source, provide a link to the Creative Commons license, and indicate if changes were made. The images or other third party material in this article are included in the article's Creative Commons license, unless indicated otherwise in a credit line to the material. If material is not included in the article's Creative Commons license and your intended use is not permitted by statutory regulation or exceeds the permitted use, you will need to obtain permission directly from the copyright holder. To view a copy of this license, visit http://creativecommons.org/ licenses/by/4.0/.

(c) The Author(s) 2019 